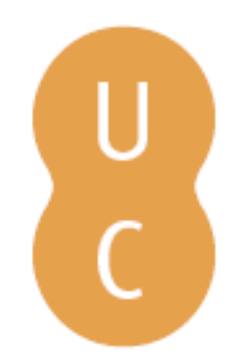

\title{
nombalina
}

\section{Ubi sunt tua tela cupido?}
Autor(es):
Deswarte-Rosa, Sylvie
Publicado por: Imprensa da Universidade de Coimbra
URL persistente:
URI:http://hdl.handle.net/10316.2/38959
DOI:
DOI:http://dx.doi.org/10.14195/978-989-26-0395-7_13

Accessed : $\quad$ 26-Apr-2023 11:17:32

A navegação consulta e descarregamento dos títulos inseridos nas Bibliotecas Digitais UC Digitalis, UC Pombalina e UC Impactum, pressupõem a aceitação plena e sem reservas dos Termos e Condições de Uso destas Bibliotecas Digitais, disponíveis em https://digitalis.uc.pt/pt-pt/termos.

Conforme exposto nos referidos Termos e Condições de Uso, o descarregamento de títulos de acesso restrito requer uma licença válida de autorização devendo o utilizador aceder ao(s) documento(s) a partir de um endereço de IP da instituição detentora da supramencionada licença.

Ao utilizador é apenas permitido o descarregamento para uso pessoal, pelo que o emprego do(s) título(s) descarregado(s) para outro fim, designadamente comercial, carece de autorização do respetivo autor ou editor da obra.

Na medida em que todas as obras da UC Digitalis se encontram protegidas pelo Código do Direito de Autor e Direitos Conexos e demais legislação aplicável, toda a cópia, parcial ou total, deste documento, nos casos em que é legalmente admitida, deverá conter ou fazer-se acompanhar por este aviso.

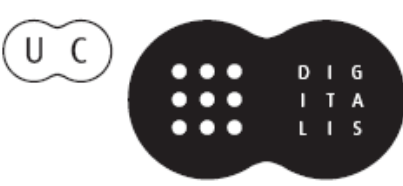


Nair de Nazaré Castro Soares

Santiago López Moreda

Coordenação

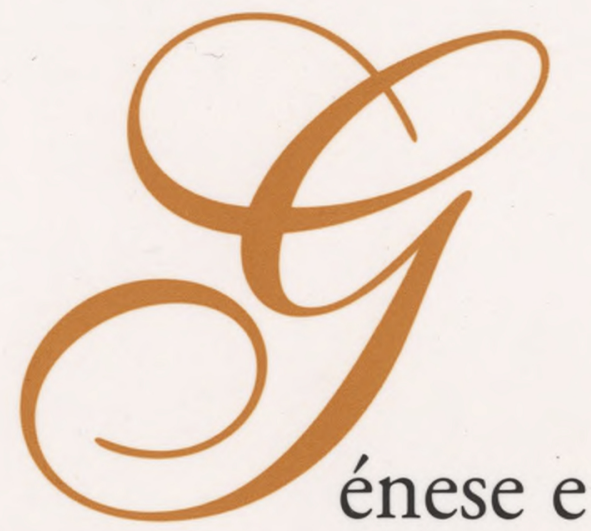

Consolidação da Ideia de Europa

Vol. IV

Idade Média e Renascimento

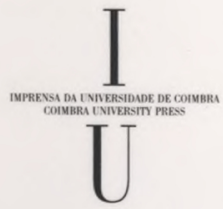

- COIMBra 2009 


\title{
UBI SUNT TUA TELA CUPIDO?
}

\author{
SYLVIE DESWARTE-ROSA \\ (CNRS - Lyon)
}

À la fin des De Aetatibus Mundi Imagines, Francisco de Holanda place ses deux images en diptyque de l'Ange du Seigneur et d'Aphrodite et Eros, chef-d'œuvre de sa jeunesse, évocation de la Fin du Monde (fig. 1, 2). Ainsi s'achève l'Histoire du Monde, suite à l'Apocalypse, prophétie de toute cette histoire. Holanda ne rajoutera plus à son livre, en manière de colophon, que l'image emblématique finale où il se portraiture, offrant ses De Aetatibus Mundi Imagines à la Malice du Temps sous la présidence des trois Vertus théologales. On est dans les années 1580 , peu avant sa mort, lorsqu'il termine son œuvre commencée à Évora, en 1545.

Ce diptyque de la Fin du Monde remonte à l'année 1545, date inscrite au bas des marches du sépulcre d'Aphrodite et Eros. Nous n'avions pas détecté cette date, à peine visible, en 1983 lors de notre première étude ${ }^{1}$, mais nous avions cependant daté ce diptyque de cette année, car tout l'indiquait : le papier à l'arbalète des années 1540 , la formule des grandes images pleines pages tout en couleurs comme dans ses images de la Création du Monde, la thématique même de l'Ange et de Vénus et l'Amour qui se retrouve dans son traité $D a$ Pintura Antigua qu'il rédige dans ces années 1540, au retour de son voyage en Italie, et surtout l'inventivité et l'audace juvénile de ces images. Ce diptyque a donc été dessiné et mis en couleur en 1545 à Évora, probablement durant ce mois d'août où Holanda commence son œuvre avec les trois premières images

'Sylvie Deswarte, «Les "De Aetatibus Mundi Imagines" de Francisco de Holanda», Monuments et Mémoires Eugène Piot, 66, Paris, P.U.F, 1983, p. 67-190 ; Id., As Imagens das Idades do Mundo de Francisco de Holanda, Lisbonne, Imprensa Nacional - Casa da Moeda, 1987 [traduction en portugais, revue et complétée]. Nous sommes revenue à ces images à l'occasion du livre d'étude accompagnant l'édition facsimilée des De Aetatibus Mundi Imagines (Barcelone, Bibliogemma, 2008). Une édition plus complète et en français devrait paraître à Genève chez Droz. 
de la Création du Monde. Mois d'août torride où il travaille à l'ombre des persiennes ou dans la fraîcheur de la chapelle-fontaine de la casa de campo de Valbom d'André de Resende.

Après tant d'images à la plume et au lavis, tout en délicatesse, de sa maturité et de sa vieillesse, ces deux grandes images de sa jeunesse, à l'atmosphère tout autre, forment un contraste violent dans ce retour abrupt à la couleur. On se trouve rejeté quarante ans en arrière, à Évora où Holanda travaille avec jubilation, animé d'une créativité intense, sous l'œil bienveillant d'André de Resende.

\section{L'ANGELVS DOMINI}

Placé après la Jérusalem céleste, l'Angelus Domini renvoie certes à l'épilogue de l'Apocalypse: «Moi, Jésus, j'ai envoyé mon Ange publier chez vous ces révélations...» (Ap. 22, 16), comme il est dit dans l'Ordem do Livro: " ê hum anjo do Apocalypsi como se nelle pinta». L'enchaînement des images se fait ainsi sans encombre, en dépit de leur disparité.

L'Ange du Seigneur est cependant, surtout et avant tout, le pendant d'Aphrodite et Eros. Il est l'Amour céleste qui l'emporte sur l'Amour profane, triomphe de la lumière céleste face à la nuit terrestre. Holanda a réfléchi sur ce thème, partant notamment d'un des emblèmes d'Alciat, Anteros, quid est Amor virtutis $^{2}$ (fig. 10,11 ). Il en a extrait les mots Ubi sunt tua tella Cupido, qu'il inscrit près du squelette de l'Amour dans l'image d'Aphrodite et Eros. Anteros, qui est l'Amour de la Vertu, a triomphé de Cupidon, l'Amour charnel. Telle est bien la clef emblématique de ce diptyque. Cependant, dans ces deux grandes images tout en couleurs, hors du commun, il est certes difficile de reconnaître la source emblématique à l'arrière-plan. On est bien loin de la petite vignette gravée du Liber Emblematum d'Alciat où Anteros, coiffé de la couronne de la Sagesse, tresse quatre couronnes, référence aux quatre vertus cardinales.

L'Ange du Seigneur, apparition flamboyante dans l'azur du ciel, surgi des nuées rosées dans un rougeoiement d'éclats de lumière sortant de ses mains, de ses pieds, de son sexe et de sa poitrine, est descendu de la sphère divine, comme nimbé du triangle de la Trinité. On retrouve ainsi, à l'arrière de l'Ange, le thème trinitaire du triangle dans le cercle, si important dans ces années 1540 ,

${ }^{2}$ Emblemata Andreae Alciati jurisconsulti clarissimi, Lyon, Guillaume Roville, 1548, p. 88 "Amor virtutis», gravure de Pierre Eskrich, emblème présent dès l'édition de 1531 (BM Lyon, Res 389 507). Voir le commentaire de Francisco Sanchez el Brocense dans l'éditionde Lyon, Guillaume Rouillé 1573, Emblème CIX, p. 327-3129. 
dessiné ici à main levée et non plus à la règle et au compas comme dans l'image du Premier Jour. On retrouve également l'harmonie de couleurs bleu, oranger et violine des images de la Création du monde. Cette figure flamboyante de l'Angelus Domini à la double paire d'ailes violines, image sublime de l'Amour céleste, forme contraste avec l'image nocturne et monochrome, quasi un chiaroscuro, d'Aphrodite et Eros, l'Amour terrestre.

C'est le premier Ange que Holanda a peint, le premier et le plus beau de tous. Francisco est alors tout à la lecture des Hiérarchies Célestes de Dionysos l'Aréopagite, auteur cher à l'infant $\mathrm{D}$. Luís, en vue de l'écriture de son chapitre 28 «Da pintura das imagens invesiveis». Il est en effet en train de rédiger avec ardeur son traité Da Pintura Antigua, qu'il achèvera en 1548. Il décrit au premier chapitre, «Como Deus foi pintor», comment Dieu, «com lux e dia, cor perfeitissima», "matizou elle as imagens encarecidas dos angelicos tronos e serafins». Holanda applique très exactement dans son image ce qu'il écrit dans son chapitre 28 sur les Anges:

Assi que, havendo de pintar as tres ordens e HIERARCHIAS dos ANGELICOS spritos, como são: ANJOS, ARCANJOS, PRINCIPADOS, POTESTADES, VERTUDES, DOMINAÇÕES, TRONOS, CHERUBINS E SERAFINS, ou pintando cada um d'estes spritos celestriaes por $\mathrm{si}$, primeiramente elles serão em formas mui proporcionadas e fremosas de meninos, ou mancebos, ou de velhos; as suas faces e vultos serão inflamados e acesos em grande fervor e amor, as mãos e os braços e os pés do mesmo ardor velocissimos e aparelhados ao mandamento e serviço divino em todo o tempo; os seus olhos enlevados e esquecidos em sua contemplação; os seus cabellos acesos como raios. Mas as suas vestiduras e ornamentos ás vezes serão de linhos alvos e castissimos, ás vezes d'especia de nuvem, e ás vezes de resplandor, ou flama; e das mãos e dos pés d'elles saião raios. As asas dobradas se podem pôr aos ANJOS, e assi mesmo nos pés por mostrarem sua presteza, mas tambem podem ser pintados sem terem asas algumas, e com tal stremidade e tão angelica, que pareça serem ANJos, como já os alguem pintou.

La confrontation de l'Angelus Domini avec ce passage de Da Pintura Antigua, comme ensuite le rapprochement d'Aphrodite et Eros avec le dessin de Vénus et l'Amour sous forme de squelettes illustrant le chapitre 18 sur l'anatomie du traité, tout cela emporta la conviction de Francisco Cordeiro Blanco, en octobre 1953, lors de sa première consultation de l'œuvre, alors anonyme, que le livre des De Aetatibus Mundi Imagines est bien une œuvre de Francisco de Holanda: «Me sentía ya pisando, no sobre arenas movedizas, sino en terreno firme», écrit-il, dans le récit de sa découverte ${ }^{3}$.

${ }^{3}$ Francisco Cordeiro Blanco, « Identification de una obra desconocida de Francisco de Holanda ", Archivo Español de Arte, t. 28, Madrid, 1955, p. 1-37, en particulier p. 14. 
L'image d'Aphrodite et Éros est ainsi décrite dans l'Ordem do Livro: «É a figura em que se torna o amor prophano e sua may Venus». Il faut la replacer dans le projet initial de l'œuvre, tel qu'il est décrit dans la Dédicace à l'Église catholique, consistant dans la Création du Monde jusqu'au Déluge, comme à la chapelle Sixtine. Aphrodite et Éros y tenait lieu déjà d'évocation de la fin du monde, correspondant à l'Imago Mortis de la Chronique de Nuremberg. Au crépuscule de sa vie, Holanda conserve cette image de sa jeunesse, fidèle à lui-même. Placée à la fin des De Aetatibus Mundi Images, cette image fait office de Memento Mori pour toute l'histoire du Monde.

La scène se passe la nuit dans le sépulcre d'Aphrodite et Éros où l'on descend par quelques marches, à la lueur des torches. Le ciel est noir et la chouette veille perchée sur le rocher. Aphrodite et Éros se dressent, droits sur leurs socles, projetant leurs ombres sur la paroi rocheuse couverte d'inscriptions gravées dans la pierre, telles des épigrammes funéraires. Les noms d'Aphrodite et Éros sont inscrits en grands caractères grecs sur la pierre, au-dessous de leurs pieds. Aphrodite est vêtue de son linceul en lambeaux, béant à la hauteur du sexe, révélant l'ossature de son bassin. La tête, encadrée de ses longs cheveux et couverte d'un voile, arbore le sourire de la mort. Les yeux fermés, elle pose telle une aveugle sa main gauche sur l'épaule de l'Amour réduit à l'état de squelette, pour qu'il lui serve de guide dans l'audelà.

Comme dans d'autres images de Holanda, tout emplies de squelettes, nous sommes face ici à la peinture de l'horrible. En effet, une œuvre peut être plaisante, non point par sa beauté, mais par sa «strana e orribile ed inaspettata invenzione», comme le note Vasari dans sa Vita di Piero di Cosimo, à propos des inventions macabres du peintre ${ }^{4}$. Les «choses horribles, pourvu qu'elles soient faites avec art, plaisent merveilleusement au goût humain». Bien le montre le plaisir que l'on éprouve à lire une tragédie, ajoute Vasari.

Aphrodite et Éros est l'image même de la Mort. Peindre «a santa imagem da Morte», figurer les os, les squelettes avec les couleurs embaumantes de la peinture, telle est bien la tâche première qui incombe au peintre, écrit Holanda dans Da Pintura Antigua $(\mathrm{I}, 18)$. Grâce au peintre, nous avons ainsi toujours

${ }^{4}$ Vasari, Vite, éd. Milanesi, IV, p. 135. Piero di Cosimo a conçu à l'occasion du Carnaval [1511 ?] un char de la Mort, hérissé de croix blanches et orné d'ossements blancs sur fond noir, de sépulcres béants d'où sortent des squelettes, "invention " qui marqua profondément les esprits. Holanda a peut-être lu cette description dans son exemplaire des Vite de Vasari. 
sous les yeux l'image de la Mort, en un perpétuel memento mori, sans avoir à fréquenter les cimetières, sans avoir à pâtir des effroyables puanteurs des corps en décomposition:

... muitas vezes o Bom Pintor deve pintar a santa imagem da MORTE, e os mortos, que muito vale a pintura e mui pouco fóra d'ella. Nem que prova mais suave pode fazer de si a pintura, que mostrar-nos aquelas cousas muito limpamente com cheirosas colores pintadas, que não podiamos ver, comprindo-nos tanto n'esta vida, señao n'um adro, ou cimiterio entre o abominavel cheiro dos finados, e entre vermens e ossos de corrução, para podéremos star contemplando, ou n'um livro, ou n'um purgaminho, ou tavoa, e téremos sempre presente o em que havemos de parar, de nenhuma outra maneira possivel melhor que na imagem da pintura! ${ }^{5}$

Aphrodite et Éros est, comme Rome déchue dans le livre des Antigualhas, une de ces images chargées de sens, aux échos multiples, que l'on peut lire de diverses façons. Elle est en fait une superposition et condensation d'images que l'on peut faire ressurgir comme dans un fondu-enchainé : la statue de la Vénus Felix du Cortile delle statue au Belvedère; la tabula anatomica de son traité Da Pintura Antigua, montrant les deux squelettes de Vénus et l'Amour, un grand et un petit; le grand dessin de la Mort en chiaroscuro de la maison de Michel-Ange; la Belle Anne, peinture du roi René à Avignon, le tout condensé en une image sublime de Memento Mori universel.

\section{LA VENUS FELIX}

On reconnaît sans mal derrière l'image d'Aphrodite et Éros le célèbre groupe sculpté de la Vénus Felix du Cortile delle Statue du Belvédère ${ }^{6}$ (fig. 3). Holanda en a peut-être fait un dessin, non conservé dans son livre des Antigualhas, lorsqu'il dessine systématiquement les statues antiques du Cortile delle statue au Belvedère : la «Cléopâtre» (f. 8v); l'Apollon (f. 9); le Laocoon (f. 9v); le Mercure (f. 29r); la «Venvs ex Balneo exiens» (f. 31); le Nil (f. 50).

Les statues antiques de Rome ont inspiré une intense activité poétique durant la première moitié du $\mathrm{XVI}^{\mathrm{e}}$ siècle. On aimait à les faire parler, «Apollo

\footnotetext{
${ }^{5}$ Francisco de Holanda, Da Pintura Antigua, I, 18, ed. A. González Garcia, Lisbonne, 1984 , p. 108-109.

Musées du Vatican, Museo Pio Clementino. Walter Amelung, Die Sculturen des Vaticanischen Museum, vol. II, Berlin, 1908, p. 112-115, $\mathrm{n}^{\circ}$ 42. Voir Sylvie Deswarte-Rosa, "Francisco de Holanda et le Cortile di Belvedere ", in Il Cortile delle statue. Der Statuenhof des Belvedere im Vatikan. Akten des internationalen Kongresses, Rome, 1992, ed. Matthias Winner, Bernard Andreae, Carlo Pietrangeli, Mayence, Philipp von Zabern, Rome, 1998, p. 389-410.
} 
loquitur», «Laocoon loquitur», etc. La plus célèbre de ces Statue parlanti est le Pasquinus que dessine Francisco de Holanda dans ses Antigualhas (f. 18r), entouré de Pasquinate. On faisait dialoguer Pasquinus avec Marforius, une autre de ces statues parlantes de Rome, comme le montre la gravure de Giulio Bonasone, Pasquinus et Marforius (1547) 7 .

A côté de cette production populaire autour de Pasquinus, fleurissaient dans une autre veine plus savante, les épigrammes latines que les poètes de la Curie romaine composaient sur les statues antiques. Le Laocoon, à sa découverte en 1506, a inspiré bien des poètes, notamment Jacopo Sadoleto. Evangelista Magdalena Capodiferro, disciple de Pomponio Leto, un des «verseggiatori» qui fréquente la casa d'Angelo Colocci, un proche des Portugais, et celle du cardinal Giovanni Colonna, est l'auteur sous le nom de Faustus d'épigrammes sur le Laocoon, l'Apollon, la Cléopâtre, la Louve de bronze ${ }^{8}$. Ainsi, en inscrivant des vers latins autour d'Aphrodite et Eros, Francisco de Holanda se place implicitement dans cette grande tradition littéraire.

À l'époque du séjour de Francisco de Holanda à Rome sous Paul III, Eurialo d'Ascoli continue cette tradition, mais en vulgaire, publiant à Rome en 1539 les Stanze sopra le statue di Laocoonte, di Venere et d'Apollo, dédiées à Alfonso d'Avalos, Marquis del Vasto, illustrées des gravures sur bois du Laocoon, de la Vénus ex balneo, et de l'Apollon'. Eurialo d'Ascoli y traite inlassa-

${ }^{7}$ Barsch XV, p.173, n. 352 ; Vienne, Albertina, inv HB XXXV, 76; Arnold Nesselrath, «Il cortile delle Statue : luogo e storia», in Il Cortile delle statue. Der Statuenhof des Belvedere im Vatikan. Akten des internationalen Kongresses, Rome, 1992, ed. Matthias Winner, Bernard Andreae, Carlo Pietrangeli, Mayence, Philipp von Zabern, Rome, 1998, p. 1-16, en particulier p. 16 et fig. 21.

${ }^{8}$ Evangelista Magdalena Capodiferro est en relation avec les Portugais, dédiant un épigramme au roi D. Manuel et en consacrant un autre au monument de l'éléphant envoyé par ce roi à Rome. Voir Biblioteca Apostolica Vaticana, Vat. Lat. 3351, Zibaldone d'Evangelista Magdalena Capodiferro (1500-1526), f. 30 «Apollo loquitur»; f. 31v «Apollo Moecenatis»: "Qui me Praxitelis fecit nec verba dedisset...»; f. 53v: "Apud Lupa Aenea in Tarpeio»; f. 97v "Laocoon in Titi imperatoris domo repertis Julio II Pontifice Maxime»; f. $122 \mathrm{r}-\mathrm{v}$ «De Statua Celopatra»; f. 130v «Emanueli Lusitaniae Regi»; f. 136v «In Monumenta elephanta». Voir aussi Vat. Lat. 3415 et Vat. Lat. 10377 (ms sur parchemin dédié à Clément VII) où on retrouvent certaines de ces compositions poétiques, magnifiquement calligraphiées. Voir Oreste Tommasini, «Evangelista Maddaleni de' Capodiferro accademico... e storico Evangelista Magdalena Capodiferron, Atti della R. Accademia dei Lincei, 1892, Série Quarta, vol. X, Rome, 1893, p. 3-20; Vincenzo Farinella, Archeologia e Pittura a Roma tra Quattocento e Cinquecento. Il caso di Jacopo Ripanda, Turin, 1992, p. 36-338, 92-94.

${ }_{9}^{9}$ Stanze d'Eurialo d'Ascoli sopra le statue di Laocoonte, di Venere et d'Apollo, al gran Marchese del Vasto [Roma, M. Valerio Dorico e Luigi fratelli, 1539] (B.A.V.: Capponi V 847; Rossiana III 1018). 
blement du même thème, comment l'art dépasse la Nature («quanto può l'arte più che la Natura»). Il interpelle le sculpteur : "Scultor maraviglioso [...] perché non facesti cortesia / Di dargli l'alma, la favella, e'l passo?». À cette statue de Vénus, il ne manque plus que l'âme, la parole et le mouvement pour être vivante.

Or, ce sont ces statues si vibrantes de vie, chantées par les poètes, que l'on retrouve dans les planches gravées des traités d'anatomie, réduites à l'état de squelettes, en de véritables memento mori.

\section{TABULA ANATOMICA}

Dans le manuscrit original, aujourd'hui perdu, de Da Pintura Antigua (1548), au chapitre 18 sur l'anatomie, Francisco de Holanda a dessiné sur une pleine page deux squelettes, dûment identifiés comme étant Vénus et l'Amour. À défaut de reproduire les dessins dans sa copie du manuscrit, Monsenhor Ferreira Gordo en donne une description ${ }^{10}$ :

Segue-se na pagina seguente dois esqueletros, um grande e outro pequeno. $\mathrm{O}$ grande tinha de baixo a inscrição seguinte: "Venvs" e o pequeno "Amor". No reverso estava o retrato d'um corpo sem pele, tendo este letreiro: "Divus Bartolomeus".

Dans ce dessin des squelettes de Vénus et l'Amour, Holanda suit clairement la tradition des tabulae anatomicae d'André Vesale dans son Humani corporis fabrica, élaboré à Padoue entre 1539 et 1542 et publié à Bâle chez Oporino en 1543, grandes planches gravées sur bois par le Flamand Jean de Calcar, de l'atelier de Titien. S'adressant aux artistes autant qu'aux médecins, les squelettes ou écorchés du traité de Vésale prennent la pose bien reconnaissable de figures de peintures célèbres ou de statues antiques en de véritables «anatomies posées» (André Chastel) ${ }^{11}$. L'écorché se dresse sur un paysage de ruines telle une statue antique. Des fragments de statues antiques, des torses aux bras et aux jambes brisées révèlent leurs viscères. L'étude conjointe de l'anatomie et des fragments antiques étaient en effet la base de l'éducation artistique comme le montre la gravure d'Enea Vico de l'Accademia de Baccio Bandinelli à Florence en 1550 où le sol est jonché au premier plan de fragments antiques et d'ossements.

${ }^{10}$ Lisboa, Academia das Ciencias, Ms Azul 650: Francisco de Holanda, Da Pintura Antigua, f. 38 r, copie exécutée en 1790 à Madrid sous la direction de Monsenhor Joaquim José Ferreira Gordo, du manuscrit original en portugais, aujourd'hui perdu.

"André Chastel, "L'anatomie artistique et le sentiment de la mort», Médecine de France, 175, 1966, p. 17-32, en particulier p. 23. 
Au verso du dessin de Vénus et l'Amour, on trouvait le «Divus Bartolomeus» en écorché, référence directe au Jugement Dernier de la Sixtine où Michel-Ange s'est représenté sous les traits d'un «San Bartolomeo bellissimo, il quale mostra la pelle scorticata» ${ }^{12}$. Ce Divus Bartolomeus est aussi un hommage rendu à la pratique de l'anatomie par Michel-Ange que Holanda évoque longuement dans son chapitre sur l'anatomie, rapportant sa fabrication de figures de cire, fait relaté par aucune autre source.

Dans le traité d'anatomie de Juan Valverde de Hamusco, Historia de la composicion del cuerpo humano, publié à Rome par Antonio Salamanca et Antonio Lafreri en $1556^{13}$, on trouve, à côté de planches tirées de Vésale, deux planches anatomiques, gravées sur cuivre, avec précisément les mêmes sujets que les dessins de Holanda, d'une part une Vénus sortant du bain, et surtout un écorché prenant l'aspect de saint Bartholomé tenant sa peau (Tab. Primera del Lib. segundo) (fig. 4). Si, comme le déclare Valverde, la plupart des gravures de son traité reprennent celles du traité de Vésale, la gravure de la figure de l'écorché en saint Bartholomé est nouvelle. Vicente Carducho dans ses Dialogos de la pintura en 1633 attribue les gravures de ce traité d'anatomie à "l'insigne" Gaspar Becerra (Baeza, vers 1520 - Madrid, 1568) ${ }^{14}$. Quant à Francisco Pacheco dans l'Arte de la Pintura (publié à titre posthume en 1649), il parle à son tour de «nuestro valiente español Gaspar Becerra que hizo tan grand demonstración de lo que sabía de músculos (como singular imitador de Miguel Angel) en el libro de Anatomia de Valverde» ${ }^{15}$.

${ }^{12}$ Vasari, Le Vite, éd. Milanesi, VII, p. 333.

${ }^{13}$ Juan de Valverde de Hamusco, Historia de la composición del cuerpo humano escrita por Ioan de Valverde de Hamusco, Rome, Antonio Salamanca y Antonio Lafrerii, 1556. A la fin: «Imprimiose la presente obra en Roma en casa de Antonio Blado Impressor de su Santidad. Anõ de MDLVI». Dédicace à «Don Fray Ioan de Toledo, cardenal y arçobispo de Santiago", daté et signé Rome, 13 septembre 1554; privilège d'impression du pape Paul IV, le 4 mai 1556 (BN, Madrid, Raros 4731). Ce traité fut traduit en italien en 1559, alors dédié à Philippe II et en latin en 1589, dans une traduction du fils de son maitre Realdo Colombo, Michele Colombo. Sur Valverde, cf. J. M. López Piñero, Ciência y técnica en la sociedad española de los siglos XVI y XVII, Barcelone, 1979, p. 325-330 .

${ }^{14}$ Vicente Carducho, Dialogos de la Pintura, su Defensa, Origen, Essencia, Definicion, Modos y Diferencias, Madrid, Francisco Martinez, 1633 ; éd. de Francisco Calvo Serraller, Madrid, 1979, p. 26 : «Leí libros doctos en esta facultad, y en las demas que componen un perfecto Pintor. Para la Notomía, el Vexalio, Autor Frances; cuyos dibujos excelentemente hizo Juan Calksux, Frances, y los del Valverde Español, el insigne Bezerra, ambos siguiendo casi en todo lo uno, $\mathrm{y}$ en lo otro al Vexalio ".

${ }^{15}$ Francis Pacheco, El Arte de la Pintura, ed. Sánchez Cantón, I, pp. 369 et 372; ed. Bonaventura Bassegoda i Hugas, Madrid, 1990, pp. 349-350. 
Gaspar Becerra, à Rome entre 1544 et 1557, appartient, comme Francisco de Holanda, à l'orbite de Michel-Ange, bien qu'aucun document ne le prouve ${ }^{16}$. La présence du Saint Barthélémy en écorché dans le chapitre sur l'anatomie de Francisco de Holanda (1548) comme dans la Tabula anatomica de Gaspar Becerra, gravée sur cuivre par Nicolas Beatricet, dans le traité de Juan de Valverde (1556) est troublante. Après Holanda et indépendamment de lui, Juan de Valverde et Gaspar Becerra, qui n'ont pu avoir connaissance du traité portugais resté manuscrit ${ }^{17}$, ont produit la même planche anatomique du Divus Bartolomeus, inspiré par la figure du saint dans le Jugement dernier de Michel-Ange.

La planche du Divus Bartolomeus est chez Holanda, comme chez Valverde-Becerra, un tribut rendu à Michel-Ange, grand praticien de l'anatomie et de la dissection.

Néanmoins, allant plus loin, on peut se demander s'il n'y aurait pas dans cette illustration du saint Bartholomé la reprise d'une planche anatomique de Michel-Ange lui-même ou d'un dessin du maître. Michel-Ange, on le sait, a réalisé de nombreux dessins anatomiques et à l'époque où Juan de Valverde est à Rome, il travaillait aux planches qui devaient illustrer le traité d'anatomie de son ami le médecin Realdo Colombo, maître de Valverde, avec lequel il pratiquait la dissection à Santa Agata dei Goti en 1548. Mais le traité de Realdo Colombo, De Re Anatomica (Venise, Nicolò Bevilacqua, 1559) (fig. 5), publié à titre posthume par les soins de son fils, ne comporte d'autre illustration que le frontispice gravé ${ }^{18}$. Seule la présence d'un jeune artiste dessinant pendant la leçon d'anatomie rappelle cette collaboration entre artistes et médecins.

On peut cependant supposer l'existence d'une série de planches anatomiques de la main de Michel-Ange, faites d'abord indépendamment de ce projet de traité puis regroupées et complétées dans cette perspective. Les deux dessins de Vénus et l'amour et du Divus Bartolomeus illustrant le chapitre de Holanda sur l'anatomie pourraient bien être la dérivation de dessins de Michel-Ange, comme le suggère David Summers qui croit y reconnaître l'ironie caractéristi-

16 Sur Gaspar Becerra, voir J. J. Martín González, «Precisiones sobre Gaspar Becerra", Archivo Español de Arte, 42, 1962, p. 327-356 ; Carmen García-Frías Checa, Gaspar Becerra y las pinturas de la Torre de la Reina del Palacio de El Pardo. Una nueva lectura tras su restauración, cat. expo. Madrid, Patrimonio Nacional, 2005.

${ }^{17}$ Gaspar Becerra put tout au plus avoir accès au traité de Holanda en Espagne même, à son retour de Rome. Il rentre d'Italie entre 1556, date de son mariage à Rome avec Paula Velázquez, et 1558 , date du contrat de son retable de la cathédrale d'Astorga, premier document connu de sa présence en Espagne.

${ }^{18}$ Il en existe un exemplaire à la Biblioteca Pública de Évora, Séc. XVI 4256. 
que du maitre ${ }^{19}$. Pour l'illustration du traité de Juan de Valverde qui reprend en grande partie les planches de Vésale, Becerra, s'inspirerait à son tour des dessins de Michel-Ange pour la création de nouvelles planches ${ }^{20}$.

La figure du Saint Bartholomé en écorché du traité de Valverde a frappé particulièrement les esprits et a souvent été reprise dans les traités d'anatomie ${ }^{21}$. Elle le fut aussi par les artistes. On trouve ainsi une statue de marbre de St Bartholomé en écorché (v. 1562) de Marco d'Agrate (actif à Milan en 1540v.1572) au Duomo de Milan, ainsi que son modelo de terracotta au Musée du Duomo $^{22}$ (fig. 6), sans doute inspirée par cette planche, alors que la tradition y voyait une reprise d'un modèle de Michel-Ange lui-même ${ }^{23}$.

\section{«CAUCHEMARS ANATOMIQUES»}

La pratique de l'anatomie est profondément pénétrée d'un sentiment de la mort et de memento mori. Le grand squelette dressé de la Mort, tenant la faux, préside ainsi à la séance de dissection et leçon d'anatomie d'André Vésale,

${ }^{19}$ David Summers, Michelangelo and the Language of Art, Princeton, 1981, Part II, chap. 13 "Anatomy ", p. 404: "Also, Francisco de Hollanda illustrated his chapters on anatomy with two skeletons, one large and one small, a Venus and Cupid, as well as flayed man, St Bartholomew. These themes at once suggest Michelangelo, and the irony of a fleshless Venus and Cupid, or an ecorché of the saint with whose skin Michelangelo identified his own image may provide some hint of the flavor of Michelangelo's projected anatomical illustrations ».

${ }^{20}$ Selon H. Cushing (A Bio-Bibliography of Andreas Vesalius, New York, 1943, p. 146) et Loris Premuda (Storia delliconografia anatomica, Milan, 1957, p. 142-144), Becerra se serait simplement inspiré du St Bartolomé du Jugement Dernier, en prenant l'idée, sans en être une dérivation littérale, n'y voyant rien de particulièrement michelangélesque.

${ }^{21}$ Pour la descendance de l'écorché de Valverde jusqu'en 1728, cf. Lola Szladitis, "The Influence of Michelangelo on Some Anatomical Illustrations », Journal of the History of Medicine and Allied Sciences, 1954, p. 420-427.

${ }^{22}$ Relevé par H. Cushing, A Bio-Biography of Andreas Vesalius, New York, 1943, p. 146, qui y voit un antécédent et non une descendance de l'illustration du traité de Valverde. Selon L. Price Amerson, The Problem of the Ecorché: A Catalogue Raisonné of Models and Statuettes from the Sixteenth Century and Later Periods, Pennsylvania State University, Thesis in Art History, 1975 (University Microfilms International ann Arbor, Michigan, USA), la statue de marbre et le modèle de terre cuite de Marco d' Agrate seraient la dérivation des planches anatomiques et n'en seraient pas le modèle comme le veut Lola Szladits.

${ }^{23}$ Cette tradition remonte à la fin du XVII ${ }^{\mathrm{e}}$ siècle, comme le note Price Amerson (op. cit., p. 88), au texte de Florent Le Comte, Cabinet des singularitez d'architecture, peinture, sculpture, Paris, 1699. Ce modèle de Michel-Ange est référé dans la Collection Von Praun à Nuremberg au XVIII ${ }^{\mathrm{c}}$ siècle (voir Christoph T. Murr, Description du Cabinet de Monsieur Paul Von Pravn à Nüremberg, Nüremberg, 1797, p. 241 n. 43. 
figurée au frontispice gravé de son Humani corporis fabrica d'André Vesale (Bâle, Oporino, 1543).

Les séances de dissections, plus ou moins clandestines, souvent nocturnes, suscitent l'effroi et le dégoût. Léonard de Vinci évoque, dans ses notes sur la pratique de l'anatomie, la compagnie horrible, et difficilement supportable, des cadavres :

E se tu avrai l'amore a tal cosa, tu sarai forse impedito dallo stomaco, e se questo non ti impedisce, tu sarai forse inpedito dalla paura coll'abitare nelle tempi notturni in compagnia di tali morti squartati e scorticati e spaventevoli a vederli ${ }^{24}$

Les scènes de déterrements de cadavres, la nuit, dans les cimetières, ont inspiré aux artistes de la Renaissance des images de "cauchemars anatomiques ", comme les appelle André Chastel ${ }^{25}$. Holanda a dans sa collection d'estampes un de ces " cauchemars ", une gravure d'Agostino Veneziano qu'il évoque dans sa liste des Famosos entalhadores de lamina de cobre: "Augustinho Venetto foi mui arrezoado, que fez as mortes[...] ». Cette gravure saisissante d'après un dessin de Rosso conservé aux Offices, montre une scène nocturne dans un cimetière, dominée par le grand squelette ailé de la Mort, rassemblement plein d'effroi et d'agitation autour d'un squelette qu'on retire du sol (fig. 7). Holanda a emprunté à cette gravure le squelette ailé de la Mort dans son image du Triomphe de la Mort des De Aetatibus Mundis (f. 68r).

La figuration du squelette est traditionnellement associée à la mor ${ }^{26}$. C'est le gisant médiéval, la danse macabre, le squelette figuré dans les livres d'heures, représenté longtemps sans aucun souci de vérité anatomique. Mais au $\mathrm{XVI}^{\mathrm{e}}$ siècle, pour peindre le squelette de la Mort, comme le souligne Holanda, il faut une bonne connaissance de l'anatomie, car on ne se contente plus des figurations approximatives de l'époque médiévale. Le squelette de la Mort est ainsi réinterprété à l'aune du savoir anatomique. De même qu'il ne s'agit pas pour Holanda de dessiner des mapamundi de fantaisie, il faut de l'exactitude dans la figuration du squelette de la Mort.

Holanda a ainsi dessiné maintes fois le squelette de la Mort dans plusieurs de ses images des De Aetatibus Mundi, rodant, entre Adam et Ève, au pied de

${ }^{24}$ Windsor 19070b ; voir Jean Paul Richter, The Literary Words of Leonardo da

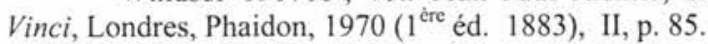

${ }_{25}$ André Chastel, «L'anatomie artistique et le sentiment de la mort », Médecine de France, 175, 1966, p. 17-32, en particulier p. 22.

${ }_{26}$ André Chastel, «L'anatomie artistique et le sentiment de la mort », Médecine de France, 175,1966 , p. $17-32$ 
l'Arbre lors de La Faute (f. 9r), enserrant le globe du monde sous l'image de La Réprimande (f. 9v), tombant tête la première dans La Descente aux Limbes (f. 52r), brandissant la faux dans son image du Triomphe de la Mort (f. 68r), gisant au sol devant les roues du char du Temps (f. 69r), chevauchant un des quatre chevaux de l'Apocalypse (f. 73r) et précipitée dans la bouche de l'Enfer dans la $X X^{e}$ Vision de l'Apocalypse (f. 84). Ce sont enfin les squelettes surgissant de la terre au jour de la Résurrection des corps (f. 68v) selon la prophétie d'Ezéchiel. Enfin Holanda peint les deux cadavres d'Aphrodite et Éros, en un ultime memento mori (f. 88r).

\section{LE DESSIN DE LA MORT EN CHIAROSCURO DE MICHEL-ANGE}

Holanda a peut-être hérité de Michel-Ange ce goût du macabre et du memento mori. Auprès de lui, il a appris que le premier devoir du peintre est de peindre la Mort. Au milieu de l'escalier de la maison de Michel-Ange, on trouvait le dessin du squelette de la Mort en chiaroscuro, debout, portant sur ses épaules un cercueil. Dans une note au madrigal traitant de l'Amour et de la Mort de Michel-Ange (Girardi 110), son neveu Michelangelo il Giovane écrit ${ }^{27}$ :

Disse Bernardo [Buontalenti] che Mich. ${ }^{\text {lo }}$ aveva a mezza la scala in casa sua in Roma dipinta una mo[rte] disegnata uno scheletro di Morte di chiaro scuro, ritto con una cassa in ispalla rozza, dove era scritta "Io dico a voi c'al mondo avete dato / l'anima e 'l corpo e lo spirto 'nsieme: / in questa cassa oscura é il vostro lato".

Ce goût du Memento Mori, on le retrouve dans la harangue que MichelAnge tient dans le premier dialogue de Donato Gianotti (v.1545), reprochant à ses amis tant de réjouissances, tant de dîners :

Et vi ricordo che a voler ritrovare et godere sè medesimo, non è mestiero pigliare tante dilettationi et tante allegrezze, ma bisogna pensare alla morte. Questo pensiero è solo quello che ci fa riconoscere noi medesimi, che ci mantiene in noi uniti [...]. Et è maraviglioso l'effetto di questo pensiero della morte, il quale - distruggendo ella per natura sua tutte le cose - conserva et mantiene coloro che a lei pensano, et da tutte l'humane passioni li difende; la quale cosa io mi ricordo haver già assai acconciamente

${ }^{27}$ Archives Buonarotti, Florence, Codice XV des Rime de Michel-Age, fol. 50b, manuscrit pour l'édition de 1623 de la main de son neveu Michelangelo il Giovane; Karl Frey, Die Dichtungen des Michelagniolo Buonarroti, herausgegeben und mit Kritischen Apparate, Berlin, 1897, cxxxvii; Enzo Noé Girardi, éd., Michelangliolo Buonarroti, Rime, con varianti, apparato, nota filologica, Bari, 1960, $\mathrm{n}^{\circ} 110$. 
accennato in un mio madrialetto, nel quale, ragionando d'Amore, conchiusi che da lui niuna altra cosa meglio che il pensierdella morte ci difende ${ }^{28}$.

Suit le madrigal de Michel-Ange ${ }^{29}$ sur la pensée de la mort, seule protection efficace contre l'amour. Le pouvoir de Vénus et de l'Amour est déjoué par leur propre image sous forme de squelettes:

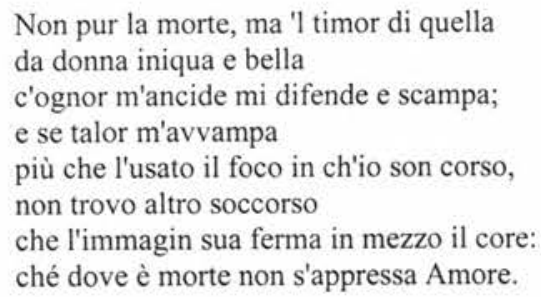

Par-delà le groupe sculpté de la Vénus Félix, par-delà la Tabula Anatomica de Vénus et l'Amour illustrant le chapitre sur l'anatomie de Da Pintura Antigua, par-delà le chiaroscuro de la Mort de la maison de Michel-Ange, Aphrodite et Éros nous conserve également le souvenir du célèbre tableau de la Belle Anne, peint à la détrempe par le roi René au couvent des Célestins à Avignon. Dans ses études sur le roi René, Otto Pächt fut le premier à rapprocher l'image d'Aphrodite et Eros de la peinture de la Belle Anne, concluant:

En vérité, l'artiste a laissé jouer sa fantaisie en transposant l'idée du transi du gothique tardif dans le domaine de l'art funéraire païen. Nous sommes en face d'Aphrodite et d'Eros, les dieux de la beauté et de l'amour, rendus comme cadavres affreux et répugnants. Au-dessus de la tête d'Eros on peut lire, gravés dans le rocher, les mots: "Nunc scio quid sit amor", un "Mortifiement" comme sujet de la mythologie classique. La source d'inspiration ne peut être autre que le tableau de la Belle Anne de René, décrit par Francisco d'Ollanda. [...] La composition énigmatique de Francisco d'Ollanda peut donc être estimée comme tribut à l'imagination artistique du roi René $e^{30}$.

${ }^{28}$ Donato Giannotti, De' giorni che Dante consumò nel cercare l'Inferno e 'l Purgatorio, ed. de D. R. de Campos, Florence, 1939, p. 69.

${ }^{29}$ Michelangelo, Rime, ed. Girardi, ${ }^{\circ} 127$.

${ }^{30}$ Otto Pächt, « Dévotion du roi René pour sainte Marie-Madeleine et le sanctuaire de Saint-Maximin ", Chronique méridionale. Arts du Moyen Age et de la Renaissance, Avignon, 1981, n 1, p. 15-27, en particulier p. 23, n. 21. Voir aussi id., «René d'Anjou. Studien I und II", Jahrbuch der Kunsthistorische Sammlungen in Wien, 69, 1973, p. 100 
Holanda est allé au couvent des Célestins à Avignon, sur le chemin de retour de son voyage en Italie, en mai-juin 1540, dans la suite de l'ambassadeur D. Pedro de Mascarenhas. Il avait dû en entendre parler peut-être déjà en Italie où l'activité picturale du roi René était connue, référée notamment par Giovanni Santi dans sa Cronaca rimata ${ }^{31}$.

Au deuxième Dialogue, dans la liste des œuvres dignes d'être vues en Italie et ailleurs, Holanda fait une longue évocation de la peinture de La Belle Anne à Avignon ${ }^{32}$ :

Mas não é de callar uma obra que vi da pintura, inda que seja fóra de Italia, em França ou Provença, na cidade de Avinhão, num mosteiro de S. Francisco, que é uma mulher morta pintada, que já fôra mui fremosa, e chamada a bella Anna ; e um rei de França que gostava de pintar et pintava (se me não engano), chamado Reynel, vindo a Avinhão, e perguntando se estava ali a bella Anna, porque desejava muito de a vêr para a tirar polo natural, e dizendo-lhe que não muito havia que era morta, fê-la el-rei desenterrar da cova, para vêr se inda nos ossos achava algum indicio de sua fremosura. Então a achou ainda ao modo antigo vestida, como se fosse viva, e os cabellos louros na cabeça ataviados, mas toda em caveira mudada a alegre fremosura do vulto, que só descuberto tinho ; e todavia assi o julgou o pintor rei por tão fremosa, que a tirou inda polo natural, com muitos versos ao redor, que a choravam e inda estão chorando. A qual obra vi naquelle lugar e me pareceu muito dina d'este.

Holanda l'évoque à nouveau, en 1571 , dans son traité De quanto serve a ciencia da pintura dans le chapitre 6 «De como el-Rei deve de saber esta ciência do desenho da pintura para seu real ornamento» (f. $45 \mathrm{v}-46 \mathrm{r}$ ):

Vi em o reino de França na cidade de Avinhão, em um mosteiro uma pintura de cores muito bem feita, a qual pintou El-Rei Reinero de França e era um Retrato da Bela Ana, que ele fez desenterrar da sepultura somente para a pintar, e assim a pintou morta como eu a vi.

Le Président de Brosses put encore voir la peinture de la Belle Anne en 1739 au couvent des Célestins et en donne la description suivante dans ses Lettres sur l'Italie:

Dans une de leurs salles, je trouvai le fameux tableau peint en détrempe par René d'Anjou, roi de Provence, leur fondateur, représentant sa maîtresse. C'est un grand squelette debout, coiffé à l'antique, à moitié couvert de son suaire, dont les vers

${ }^{31}$ Giovanni Santi, Cronaca rimata, cap. LXXXXI, vv. 23-25, fol. 214 r: «Fuor dotti in ciò: e sepper sua ragione/ Ai nostri di lo antico Re Raniero/ Depinse e a molti chiar lui se prepone». Cité par A. González Garcia dans son édition de Da Pintura Antigua (II, 2, p. 259, n. 708).

32 Da Pintura Antigua, II, 2, éd. A. González Garcia, Lisbonne, 1984, p. 259; trad. M. Denis, Madrid, 1921, p. 166. 
rongent le corps défiguré d'une manière affreuse ; sa bière est ouverte appuyée debout contre une croix de cimetière et pleine de toiles d'araignées fort bien imitées. ${ }^{33}$.

Francisco de Holanda s'inspire donc dans son Aphrodite et Éros de La Belle Anne, peinture qui a tant frappé les voyageurs. Il en reproduit l'atmosphère sépulcrale, les deux cadavres debout, et les nombreux vers de poésie inscrits sur les parois.

Avant lui, vers 1430, un enlumineur de Rouen, a peint La Belle Anne à la fin du Livre d'heures de Jacques II de Chastillon (BnF, Nouv. Acq. Lat 323, p. 545) : elle se dresse réduite à l'état de cadavre rongé par les vers, debout, coiffé d'un hennin, portant son linceul et tenant un parchemin où est inscrit la "Complainte de la Damoiselle » ${ }^{34}$ (fig. 8).

Cette image d'Aphrodite et Éros n'est pas le premier memento mori que dessine Holanda. Lors du voyage d'aller, en chemin vers la Trève de Nice, Francisco de Holanda a visité avec D. Pedro de Mascarenhas, fin avril 1538, les hauts lieux du culte de la Madeleine, la Sainte-Baume évoquée dans son image de la Madeleine pénitente (f.60v ) ainsi que Saint-Maximin. Dans son livre des Antigualhas (f. 48v), il dessine le reliquaire de Madeleine à SaintMaximin, buste de la sainte, soutenu par des anges, contenant le crâne de la sainte, normalement occulté sous un masque d'or amovible, montrant son visage dans toute sa pureté. Haut lieu de pèlerinage, on découvrait aux yeux des pélerins, derrière le masque d'or, les ossements du chef de la Madeleine.

\section{LES INSCRIPTIONS DE VERS LATINS. UN AMUSEMENT D'HUMANISTE}

Autour de La Belle Anne, on trouvait « muitos versos ao redor, que a choravam ", comme le rappelle Holanda dans son évocation de la peinture du roi René au Deuxième Dialogue. Holanda reprend l'idée dans son image et constelle la paroi rocheuse du sépulcre d'Aphrodite et Éros, de vers latins.

Dans ces inscriptions sur la paroi rocheuse éclairées par la lumière mouvante des torches, il y a également le souvenir des graffiti sur les murs de la

${ }^{33}$ Le Président de Brosses, Lettres sur l'Italie, Paris, An VII, Lettre II, vol.I, p. 23. Joaquim de Vasconcellos a relevé ce passage dans son édition viennoise des Dialogues de Rome, Francisco de Hollanda. Vier Gespräche über die Malerei geführ zu Rom 1538, Vienne, 1899 , p. 209 , n. 74.

${ }^{34}$ Voir Susie Nash, «Le Livre d'heures de Jacques II de Chastillon chef d'œuvre acquis par la BnF"), Art de l'enluminure, hors-série Art \& Métiers du Livres, n 2, Editions Faton, [2000] p. 103 (commentaire d'Albert Chatelet). Nous remercions Alexandre Parnotte pour nous avoir signalé cette enluminure. 
Domus Aurea que les artistes, séjournant à Rome, traçaient en témoignage de leur passage $\mathrm{e}^{35}$. Holanda a beaucoup fréquenté ce haut lieu de la peinture antique, y dessinant notamment la fameuse volta dorata, conservée dans son livre des Antigualhas (f. 47 bis v.- 48r).

Lorsqu'il écrit ces inscriptions sur la paroi du sépulcre d'Aphrodite et Éros, Holanda a certainement en tête les épigrammes funéraires, petites pièces de vers à la mémoire des chers disparus, qu'il a tant étudiés dans son exemplaire des Epigrammata Antiquae Urbis (Rome, J. Mazochius, 1521) (BN Lisbonne, Res. $1000 \mathrm{~A}^{1}$ ) dans la compagnie d'André de Resende. Mais au lieu d'épigrammes funéraires pleurant les défunts, il inscrit sur la paroi une série de vers des poètes élégiaques latins d'un humour grinçant. Au lieu de déploration, la dérision. On trouve ainsi des vers tirés des Bucoliques de Virgile, des Amours d'Ovide et des Élégies de Properce et de Tibulle, comme de l'Anthologie grecque. On peut lire sur la paroi à partir du haut:

\author{
NVNC.SCIO./QVID SIT.AMOR \\ Maintenant je sais /ce qu'est l'amour (Virgilio, Bucólicas, 8, 43) \\ (au-dessus de la tête d'Éros) \\ TENERA/ CVM MATRE./CVPIDO \\ Cupidon avec sa tendre mère \\ (Ovidio, Amores, I, 6, 11) \\ (entre la main d'Aphrodite et la tête d'Éros) \\ QVICVNQ.ILLE FUIT.PVERVM.QVI./PINXIT.AMOREM./ \\ NONNE.PVTAS.MIRAS.HVNC./HABVISSE. MANVS. \\ Quel que soit celui qui a peint l'amour enfant, ne trouves-tu pas qu'il a une main \\ admirable? \\ Quienquiera que fuese aquel/que pintó al amor Niño,/¿no crees que tenía/ \\ unas manos admirables? \\ (Propercio, Elegias, II, 12, 1-2) \\ (sur la paroi rocheuse à droite) \\ VBI. SVNT.TVA. TELLA./ CVPIDO. \\ Où sont tes flèches, Cupidon? \\ (Andrea Alciat, Emblematum Liber, citant l'Anthologia graeca, 16.201) \\ (sur la dalle au-dessus de l'escalier) \\ O. FORMOSE.PVER. /NIMIVM.NECREDI/COLORI./ \\ Ô bel enfant !/ Ne te fie pas trop à la couleur. \\ (Virgilio, Bucólicas, 2,17) \\ (sur la dalle au-dessus de l'escalier)
}

${ }^{35}$ Nicole Dacos, La Découverte de la Domus Aurea et la formation des grotesques à la Renaissance, Londres-Leyde, 1969; id., Roma Quanta fuit ou l'invention du paysage de ruines, Bruxelles, Maison d'Érasme-Paris, Somogy, 2004 ( $1^{\text {ère }}$ éd. italienne, Rome, Donzelli, 1995). 
LONGVS.AMOR TA/LES.CORPUS. TENV/AVIT.IN. VSUS.

Un long amour/ a affaibli mon corps/ dans ses travaux

(Ovidio, Amores, I, 6, v. 5)

(sur la dalle au-dessus de l'escalier)

EXORATA MEIS/ ILLVM./ CYTHEREA./CAMOENIS.

Vaincue par mes chants, Vénus

Vencida por mis Cantos, / Venus lo...

(Tibulle, Elegias, III, 13, v. 3)

(entre Aphrodite et l'Amour)

$\mathrm{A} \Phi \mathrm{P} \Omega \Delta \mathrm{ITH} E P \Omega \Sigma$

Afrodita Eros

(sous les pieds d' Aphrodite et d'Éros)

AT.QVONDAM.NOCE.SIMVLACHRA.QVE VANA. /TIMEBAM

Mais en d'autre temps, je craignais la nuit et ses vains fantômes

(Ovide, Amores, I, 6, v. 9)

(sous Aphrodite)

Le montage d'inscriptions est savamment élaboré. La recherche de vers qui ridiculisent Aphrodite et Éros réduits à l'état de cadavres, a dû beaucoup occuper Francisco de Holanda cet été 1545 à Évora. Holanda a sans doute demandé l'aide d'André de Resende pour l'orienter dans ses lectures. La tâche était chose facile pour un humaniste et un poète néolatin de la trempe de Resende. On assiste ainsi dans l'élaboration de cette image d'Aphrodite et Éros à un véritable travail de collaboration entre les deux hommes, une sorte d'amusement humanistes, de ludus epigraphicus, dans la meilleure tradition des horti letterari romains.

Dans cette activité poétique autour d'Aphrodite et Eros, auprès de la Fontaine aux muses de la casa de campo de Valbom, près d'Évora, d'André de Resende, Francisco de Holanda se situe dans la tradition romaine des jardins littéraires. Il ne manque que la nymphe endormie de ces jardins. Holanda a beaucoup entendu parler de ces rencontres poétiques par l'ancien ambassadeur portugais D. Miguel da Silva, lui-même poète néolatin, qui a vécu la grande époque des orti letterari à Rome, à l'époque de LéonX et de Clément VII, et par Blosio Palladio, l'ami de D. Miguel et le protecteur de Francisco à Rome, auteur de la Coryciana (Roma, 1524) et continuateur de cette tradition sous Paul III dans les Orti Blosiani de sa villa au Val d'inferno, dessinés par Baldassarre Peruzzi ${ }^{36}$.

${ }^{36}$ Sylvie Deswarte-Rosa, Il " Perfetto Cortegiano » Dom Miguel da Silva, I Rinascimenti fuori d'Italia 1, Roma, 1989, p. 33-34: «Le fontane della ninfa adormentata: Huius 
Un indice vient confirmer la part active qu'eut André de Resende dans le choix des vers. Au-dessus de l'entrée du tempietto des Muses de sa casa de campo de Valbom, fontaine et chapelle tout à la fois, est gravée une inscription composée par Resende en l'honneur de la croix qui couronnait à l'origine l'édifice ${ }^{37}$ (fig.9). Cette inscription se termine par les mots d'inspiration ovidienne «ESPECTARAQVE VANA TIME» (tu ne craindras pas les vains spectres). Or, ces mots, on les retrouve presqu'à l'identique dans l'inscription sous les pieds d'Aphrodite, tirée des Amores d'0vide (I, 6, 11): «...QVONDAM. NOCE. SIMVLACHRAS. QVEE. VANA. TIMEBAM» (Mais en d'autre temps, je craignais la nuit et ses vains fantômes) ${ }^{38}$. Une telle concordance de sources ne peut être le fait du hasard.

Enfin, on trouve la trace de cette recherche livresque menée par Francisco de Holanda dans un incunable des Disputationes Camadulenses de Cristoforo Landino, conservé à Biblioteca Pública de Évora (Inc. 571) ${ }^{39}$, sans doute un des livres de la bibliothèque d'André de Resende. Une annotation à la plume, "Quid sit amor. / Verg.», qui semble bien de la main de Francisco de Holanda, est inscrite en manchette, à côté de la définition de l'amour selon Platon. C'est un bout de vers tiré des Bucoliques de Virgile (VIII, 43), que l'on retrouve au complet, NUNC SCIO/ QUID SIT AMOR (Maintenant je sais ce qu'est l'Amour), sur la paroi sépulcrale d'Aphrodite et Éros. Nous surprenons ici Francisco de Holanda en pleine élaboration de son image, lisant les auteurs du néoplatonisme florentin qui l'ont tellement influencé dans la composition de ses premières images des De Aetatibus Mundi et dans la conception de son traité Da Pintura Antigua. Cette annotation des Disputationes Camaldulenses de Landino nous

Nynpha Loci...». Sur la Coryciana [Impressum Romae apud Ludovicum Vicentinum et Lautitium Perusinum, Mense Iulio, 1524], voir J. Ruysschaert, « Les péripéties inconnues de l'édition des "Coryciana" de 1524», Atti del Convegno di studi su Angelo Colocci, (Iesi, 1969), Iesi, 1972, p. 45-60. Sur la Villa de Blosio Palladio et les Orti blosiani, voir Daniela Pagliai, " Natura e mitologia nella villa di Blosio Palladio », in Silvia Danesi Squarzina, éd., Roma centro ideale della cultura dell' Antico nei secoli XV e XVI, Milan, 1989, p. 347-355; Maurizio Ricci, «Un contributo alla conoscenza di Lorenzo Donati allievo Senese di Baldassarre Peruzzi», Bolletino d'Arte, 91, 1995, pp. 71-94, scheda 18 et fig. 46.

37 «Flecte Genv. en signum, per quod vis victa tirani/ Antiqui atque erebi concidit Imperium / Hoc tu sive pius frontem, sive pius frontem, sive pectora signes, / Nec Lemorvm insides, espectaraqve vana timen (Plie le genoux devant le signe par lequel fut vaincue la force de l'antique tyran [le diable] et fut abattu l'empire d'Erèbe. En te signant pieusement sur le front ou sur la poitrine, tu ne craindras ni les pièges des Lémures, ni les vains spectres). Voir Sylvie Deswarte-Rosa, Ideias e Imagens em Portugal na época dos Descobrimentos. Francisco de Holanda e a teoria da arte, Lisbonne, Difel, 1992, p. 168-169.

${ }_{30}^{38}$ Alberto Rosa est l'auteur de ce rapprochement.

${ }^{39}$ Cristoforo Landino, Disputationes Camadulense, [Florence, Nicolaus de Alemannus, 1480-1490], folio 39v; Deswarte-Rosa, 1992, p. 132. Nous remercions Alberto Rosa pour l'analyse de l'exemplaire de la Biblioteca Pública de Évora (Inc. 571). 
confirme une fois de plus l'étendue des lectures de Holanda, qui ne se contente pas, comme la plupart de ses congénères, de recourir aux florilèges. Il ne craint pas d'aborder toutes sortes d'ouvrages dans les champs de la philosophie, de la théologie et de la littérature, sous l'orientation d'André de Resende et peut-être d'autres humanistes à la cour, tels les frères Sanches.

\section{LE TRIOMPHE D'ANTEROS, L'AMOUR DE LA VERTU}

Les vers inscrits autour d'Aphrodite et Éros sont tous tirés de poètes élégiaques latins, Ovide, Tibulle, Properce, à l'exception du vers Ubi sunt tua tella Cupido, provenant d'un emblème d'Alciat, repris de l'Anthologia graeca. Cet emprunt à Alciat révèle l'origine emblématique de l'image d'Aphrodite et Éros et nous donne la clef du diptyque. L'emblème d'Alciat est consacré à Antéros, id est amor virtutis (fig. 10,11). Anteros, qui est l'amour de la Vertu, demande à Cupidon: «Dic ubi sunt incurvi arcus? ubi tela Cupido?» «Cupidon, où est l'arc \& flesches dont tu tires?». Antéros, l'Amour de la Vertu, sans arc, ni flèche, ni ailes ${ }^{40}$, est coiffé de la couronne de la Sagesse et porte à son bras trois couronnes de fleurs, qui sont les vertus cardinales de la Force, de la Tempérance et de la Justice. Ces couronnes de fleurs que tresse Antéros, on les retrouve ornant la tête des Vertus dans l'image colophon où Holanda se portraiture (f. 89r). Bien des années ont passé et néanmoins, Holanda a toujours en tête la source emblématique de son diptyque et relie, par ce biais ténu, ses images les unes aux autres, en dépit des disparités de style et d'atmosphère.

\section{MEMENTO MORI UNIVERSEL}

Replacé en fin de Chronique du Monde, ce diptyque devient un Memento Mori à échelle universelle ; toute l'histoire du Monde apparaît ainsi comme une

${ }^{40}$ Dans l'édition parisienne de Chrétien Wechel (Paris, 1534), l'Amor Virtutis, figuré d'abord dans la vignette gravée avec des ailes, est dans les éditions suivantes représenté sans ailes, car il n'a ni flèches, ni ailes («ubi pennae?»). Voir Emblematum libellus, Paris, Chrétien Wechel, 1534, f. 86 Amor Virtutis, avec ailes (BNF, Paris, Z 2511); id, 1535, f. 86 Amor virtutis, sans ailes (BNF, Paris: Res Z 2515). Diego López dans sa Declaración magistral de los Emplemas de Alciato (Najera, 1615, emblema 108) donne le commentaire suivant: «Ay dos Cupidos... el uno divino, y celestial el qual infunde los buenos, y santos desseos, y es contrario de Cupido el deshonesto, porque infunde a los hombres los tropes, y deshonestos desseos. El otro por lo contrario dispierta [sic] al hombre a contemplar las cosas divinas, $\mathrm{y}$ celestiales, $\mathrm{y}$ les da toda la virtud, $\mathrm{y}$ honestidad. $\mathrm{Y}$ deste amor santo, $\mathrm{y}$ honesto se trata aquí... Lo qual significan las quatro coronas que tiene. La lque está en la cabeça significa la sabidura. las otras tres significan las tres virtudes, Fortaleza, Justicia y la Templança » 
vaste réflexion sur la mort, sur la vanité des Empires et des Âges, tous voués à la même mort. «Notre Mère la Terre est déjà bien fatiguée et épuisée», constate Holanda dans son traité (PA, II, 4). Après être passée par tous les Âges, elle ne sera plus qu'un grand corps mort. Tout périt, tout est englouti, comme ces images mêmes dont déjà se saisit la Malice du temps. Plus rien ne compte que l'éternité.

\section{L'IMAGE COLOPHON}

Francisco de Holanda termine son œuvre par une image ainsi décrite dans l'Ordem do Livro: « A ultima ê o autor com o livro das idades que ê este e a maliçia do tempo tho come.». Cette image joue ainsi le rôle de colophon à la fin du livre. Holanda rajoute cette image pour terminer son œuvre, utilisant le papier à la grappe de raisin ${ }^{41}$ qu'il a utilisé pour ses images de l'Apocalypse et que l'on retrouve dans les pages de garde. Nous sommes donc dans les années 1580, à la veille de sa mort en 1584 .

Dans cette image, Holanda se représente (fig. 12), dans un cadre ovale, livrant le livre des De Aetatibus Mundi Imagines à la Malicia Temporis figurée par un chien aux dents accérées, le tout sous la présidence de trois vertus couronnées de roses, avec au-dessous sur un table all'antica les mots FINIS. DEO., écrits en capitales à la manière d'une inscription épigraphique, encadrée par deux hedera. Il se portraiture donc, mais sans s'identifier. C'est ainsi que l'œuvre est restée longtemps anonyme jusqu'à ce qu'en 1953 Francisco Cordeiro Blanco y reconnaisse la main de Francisco de Holanda.

Francisco de Holanda aime à se représenter dans ses œuvres, le plus souvent incognito, comme Hitchcock dans ses films. On le voit tout jeune homme, âgé de vingt ans, auprès des antiques à Rome dans son livre des Antigualhas, avec Michel-Ange au pied de la Muse Melponène, statue colossale dans le cortile du Palais de la Chancellerie (Antigualhas, f. 10r), ou encore contemplant les Trophées de Mario (Antigualhas, f. 15r), enfin dessinant, couché dans l'herbe, un duel à Moncalieri (Antigualhas f. 50v), signalant exceptionnellement sa présence par l'annotation « Francisco ». Dans les De Aetatibus Mundi, nous le voyons se confessant et communiant sous l'image de la Mission des Apôtres (f. 55r). Puis à nouveau, au premier rang des élus, recevant de l'Ange le sceau divin, dans la Sexta Visio de l'Apocalypse (f. 76r). Dans cette image comme dans l'image colophon, Holanda, né en 1517, est alors dans sa soixantaine.

${ }^{41}$ Le papier au filigrane à la grappe de raisin surmontée des lettres A. F. (Briquet IV, 13160): papier de la seconde moitié du XVI ${ }^{e}$ siècle fabriqué en France: Lyon 1558-1563; Bourg-en-Bresse, 1568; Narbonne, 1575; Autun, 1580. 
Jorge de Segurado dans sa monographie sur Francisco D'Ollanda $(1970)^{42}$ a relevé la «flagrante semelhança» entre l'autoretrato de Holanda dans cette image colophon et la tête sculptée du Christ du Crucifix de marbre d'Estremoz placé au-dessus de l'autel de la Capela das onze mil virgens de l'église du Convento de Santo António à Alcácer do Sal. Ce Crucifix a été commandé par le vice-rei da Índia Don Pedro Mascarenhas, l'ancien ambassadeur portugais à Rome avec qui Francisco de Holanda est allé à Rome. La chapelle fut achevée après la mort de Mascarenhas à Goa le 23 juin 1556 par sa veuve. Segurado attribue cette sculpture à Francisco de Holanda qui se serait ainsi portraituré en Christ tout comme l'a fait Albrecht Dürer dans un Autoportrait célèbre (1500, Munich, Alte Pinakothek $)^{43}$.

Nous voyons dans cette image la confluence de deux thématiques bien connues de l'histoire du livre mettant en scène l'auteur : l'auteur présentant son œuvre achevée à son dédicataire ou commanditaire et l'auteur confronté à ses critiques.

C'est d'abord la récupération et le détournement de la scène traditionnelle de présentation de l'œuvre par son auteur, souvent agenouillé, à son dédicataire ou commanditaire, scène que l'on trouve d'ordinaire à la première page des manuscrits ou des incunables du début de l'imprimerie. Son père António de Holanda, dans l'enluminure des chroniques portugaises, représente ainsi Rui de Pina remettant au roi D. Manuel la Crónica de D. João II ou encore la Crónica do Rei D. Duarte (Lisbonne, ANTT). Francisco reprend cette scène et la détourne. Au lieu d'une demande de protection, c'est ici plutôt une scène d'abandon. De cette scène empreinte d'ordinaire du plaisir de la dédicace, de la sérénité de l'hommage rendu, du soulagement de l'œuvre achevée, Holanda en fait une scène dominée par l'angoisse, où seule la Foi le sauve. Il abandonne son œuvre à la Malice du Temps, à la vindicte publique, faisant confluer une autre thématique de l'histoire du livre, l'auteur conjurant les critiques. En effet comme écrit Francesco Priscianese, «che cosa é quella tra le cose humane che non sia sottoposta à morsi della invidia? [...] Et questo costume di mordere altri per cupidigia di gloria è vitio humano» ${ }^{44}$. De même, dans son édition

\footnotetext{
${ }^{42}$ Jorge Segurado, Francisco d'Ollanda, Lisbonne, 1970, p. 378-383 «O Cristo do relicário da Capela das onze mil virgens da igreja de Alcácer do Sal» et fig. 102-103.

${ }^{43}$ Erwin Panofșky, Albrecht Dürer, [3rd ed.], Princeton: Princeton University Press, 1948 (Ière éd. 1943), p. 43 et cat. n 50, fig. 110; tr. fr. La Vie et l'art d'Albrecht Dürer, Paris], Hazan, 1987, p. 78.

${ }^{44}$ Francesco Priscianese, Della lingua romana, Venise, Bartolomeo Zanetti da Brescia, 1540, «Francesco Priscianese al molto reverendo messer Lodovico Becci et al suo M. Luigi del Riccio».
} 
italienne des Métamorphoses d'Ovide, (Lyon, Jean de Tournes, 1559), Gabriele Symeoni se représente à la fin de l'œuvre sous les traits d'Hercule terrassant l'envie de sa massue.

La Malice du Temps est un thème récurrent dans l'œuvre de Francisco de Holanda à la fin de sa vie. On la retrouve dans son image Inigma qu'il place de la même manière, comme une sorte de colophon, à la fin du manuscrit de ses deux mémoires adressés au roi Dom Sébastien en 1571, l'un consacré aux monuments qui manquent à la ville de Lisbonne et l'autre traitant de l'Art du Dessin - Da Fábrica que falece à Cidade de Lisboa et Da Ciência do Desenho ${ }^{45}$.

La scène est présidée par trois vertus couronnées de roses et d'un nimbe doré traité en perspective. La couronne de fleurs a ainsi remplacé la colombe qui coiffait les vertus dans les images précédentes (f. 55v-56r).

Holanda, en plaçant les vertus dans le ciel, couronnées de fleurs, fait-il implicitement référence au Tableau de Cébès, une œuvre qu'il connaît bien et qu'il cite dans ses Dialogues? En effet, dans le Tableau de Cébès, la Félicité, entourée des Vertus, couronnées de fleurs, régne tout en haut dans sa citadelle isolée, à laquelle on accède par un chemin escarpé, bordé de précipices. Elle attribue une couronne de fleurs, porteuse de bonheur, à ceux qui sont parvenus jusque là et qui ont triomphé des vices ${ }^{46}$.

La couronne de fleurs est un motif récurrent chez Holanda. Il l'a trouvée dans le Tableau de Cébès, mais aussi chez Prudence, le poéte ibérique qui l'accompagne du début à la fin de l'élaboration de ses De Aetativus Mundi Imagines. Dans la Psychomachie de Prudence (v. 37) par exemple, la Foi, à la fin de son combat contre l'Idolâtrie, couronne de fleurs ses compagnons selon la gloire que chacun a acquise.

Holanda attribue une couronne de roses, ainsi qu'une couronne de laurier, à Michel-Ange dans le portrait en médaillon qu'il fait figurer au début de son livre des Antigualhas (f. 2).

Ces couronnes des vertus sont tressées par Antéros, l'Amour de la Vertu, dans l'emblème d'Alciat "Anteros, id est amor virtutis ", dont Holanda a extrait un vers pour son image d'Aphrodite et Éros. Par le biais des vertus et de leurs couronnes, Holanda crée ainsi un lien, certes ténu, quasi invisible, qu'il est seul à connaître, entre ces deux images élaborées à près de quarante ans de distance.

${ }^{45}$ Voir notre article « L'énigme d'Inigma. A propos d'une image de Francisco de Holanda ", dans L'énigmatique à la Renaissance: formes, significations, esthétiques. Colloque organisé par l'association RHR, Lyon, 7-10 septembre 2005 (sous presse).

${ }^{46}$ Nous remercions encore une fois Alexandre Parnotte qui nous a rappelé ce passage lors d'un de nos séminaires. 
On reconnaît facilement deux des vertus théologales assises dans le ciel sur les nuées, la Foi, tenant la croix aux stigmates, la Charité avec le cœur saignant. Une troisième vertu, d'interprétation plus complexe, occupe le centre de la scène, les pieds solidement posés sur le sol. Elle est vêtue d'une tunique à franges à la mode hébraïque et porte autour du cou un tore à tête d'angelot. Elle enserre du bras gauche une colonne ionique et de la main droite elle brandit un fouet à trois lanières. C'est la troisième vertu théologale, l'Espérance (SPES), selon la triade canonique SPES/ FIDES/ CHARITAS, dont nous voyons les noms inscrits sur la corolle de la Virga de Jessé (f. 37v), et que l'on retrouve dans les tondi aux bas des images de L'incrédulité de saint Thomas et de la Vocation de Pierre (f. 55v-56r). C'est la triade sans cesse invoquée par Bernardino Occhino dans ses prêches, «Viva fede, speranze e carità perfetta», que Holanda a fait sienne.

Cependant si la vertu, au centre de cette ultime image, est bien la vertu théologale de l'Espérance (SPES), elle s'appuie sur deux des vertus cardinales, la Force et la Tempérance, représentées ici par leur attribut respectif, la colonne et le fouet. On reconnaît facilement ces attributs, car on les a déjà vus associés à ces deux vertus cardinales dans les tondi sous l'image de La Descente de l'Esprit Saint (f. 57r). Le fouet, attribut de la Tempérance, se trouve aussi, on s'en souvient, entre les mains de la Providence divine au Septième Jour, qui accompagne toute l'histoire du monde (f. 11r). L'Espérance se confond ainsi avec la Providence divine. C'est elle, secondée par la Force et la Tempérance, qui vient s'interposer entre l'auteur et la Malice du Temps et veiller sur la fortune et le devenir du livre des De Aetatibus Mundi Imagines de Francisco de Holanda. C'est sur elle que Holanda, confiant, fixe son regard.

Mais, si l'on replace cette image dans le contexte des écrits théoriques de Holanda, la colonne de la Force apparaît aussi comme celle du Dessin, «sustancia e ossos das pintura ${ }^{47}$. Holanda déclare dans Da Pintura Antigua que le dessin «é a colunna desta arte»" ${ }^{48}$, cette colonne entre l'invisible et le visible, entre contemplation et création, entre l'Idée et l'œuvre achevée, cette colonne sur laquelle il s'est appuyé tout au long de la réalisation des De Aetatibus Mundi Imagines.

\footnotetext{
${ }^{47}$ Da Pintura Antigua, I, 16 «Em que consiste a força da Pintura». Sur Holanda et le dessin, voir Sylvie Deswarte-Rosa, «Tudo o que se faz em este mundo é desenhar. Francisco de Holanda entre théorie et collection», in El modelo italiano en las Artes Plásticas de la Peninsula Ibérica durante el Renacimiento, dir. Maria José Redondo. Actes du Symposium international de la Universidad de Valladolid, 16-18 octobre 2003, Valladolid, Secretariado de Publicaciones de la Universidad de Valladolid, 2004, p. 247-290, en particulier p. 251-255 «Le dessin, incarnation de l'Idée, colonne de la peinture et source de tous les arts et sciences»".

${ }^{48}$ Da Pintura Antigua, I, 44 «De todos os generos e modo do pintar», p. 198.
} 
Francisco de Holanda termine par les mots FINIS DEO écrits en littera antiqua $^{49}$, encadrés des deux feuilles de lierre ou hedera, inscrits à la manière d'une inscription sur une table de marbre entre deux têtes de lions se faisant face. C'est l'adaptation à l'antique des encadrements xylographiques qu'il trouvait dans l'Epigrammata Antiquae Urbis de Mazochius (f. XVIIIv, LXV, XLVIII). Ces têtes de lion, qu'il a copiées à Rome (Antigualhas, f. 17), Holanda les a utilisées ailleurs, les plaçant en haut des balustres de la balustrade qu'il propose pour entourer l'église de S. Sebastião à Lisbonne dans $D a F a ́$ brica que falece à Cidade de Lisboa (f. $27 \mathrm{v})$.

C'est sur ces mots Finis Deo, dans une ultime action de grâce, que Francisco de Holanda termine ainsi l'œuvre de sa vie.

${ }^{49}$ Holanda aime à terminer ses travaux pour une action de grâce. Ainsi rajoute-t-il, écrits à la plume, les mots LAVS DEO ET GLORIA à la fin de la Vie de Marie l'Egyptienne, qu'il a lue et annotée avec une attention extrême, dans son exemplaire des Vitas Patrum (BN Lisbonne, Res 198A). 
1. Francisco de Holanda, Angelus Domini, De Aetatibus Mundi Imagines, f. $87 \mathrm{v}$. Madrid, Bibliothèque nationale d'Espagne.

2. Francisco de Holanda, Aphrodite et Eros, De Aetatibus Mundi Imagines, f. $88 \mathrm{r}$. Madrid, Bibliothèque nationale d'Espagne.

3. François Perrier, Vénus Félix, gravure in Segmenta nobilium signorum et statuarum, Rome 1638, Pl. 86.

4. Gaspar Becerra, Saint Bartholomé tenant sa peau, gravure dans Juan de Valverde de Hamusco, Historia de la composición del cuerpo humano escrita por Ioan de Valverde de Hamusco, Rome, Antonio Salamanca y Antonio Lafrerii, 1556, Tab. Primera del Lib. segundo.

5. Realdo Colombo, De Re Anatomica, Venise, Nicolò Bevilacqua, 1559. Biblioteca Pública de Évora, Séc. XVI 4256.

6. Marco d'Agrate (actif à Milan en 1540-v.1572), Statue de marbre de St Bartholomé en écorché (v. 1562) au Duomo de Milan.

7. Rosso Fiorentino, Scène nocturne dans un cimetière, dessin. Florence, Galleria degli Uffizi, Gabinetto dei Disegni e delle Sampe, Inv. 14669 F

8. Enlumineur rouennais, La Belle Anne à la fin du Livre d'heures de Jacques II de Chastillon, vers 1430, Paris, BnF, Nouv. Acq. Lat 323, p. 545.

9. Chapelle-fontaine de la casa de campo de Valbom d'André de Resende. Photo S. Deswarte-Rosa.

10. Andrea Alciat, Emblème Anteros, quid est Amor virtutis, in Emblemata Andreae Alciati jurisconsulti clarissimi, Lyon, Guillaume Roville, 1548, p. 88 (avec ailes), gravure de Pierre Eskrich, BM Lyon, Res 389507.

11. Andrea Alciat, Emblème Anteros, quid est Amor virtutis, in Emblematum libellus, Paris, Chrétien Wechel, 1535, f. 86 (sans ailes), BNF, Paris: Res Z 2515.

12. Francisco de Holanda, L'auteur, sous la présidence des trois vertus théologales, livre son œuvre à la Malice du Temps, De Aetatibus Mundi Imagines, f. 89r. Madrid, Bibliothèque nationale d'Espagne. 


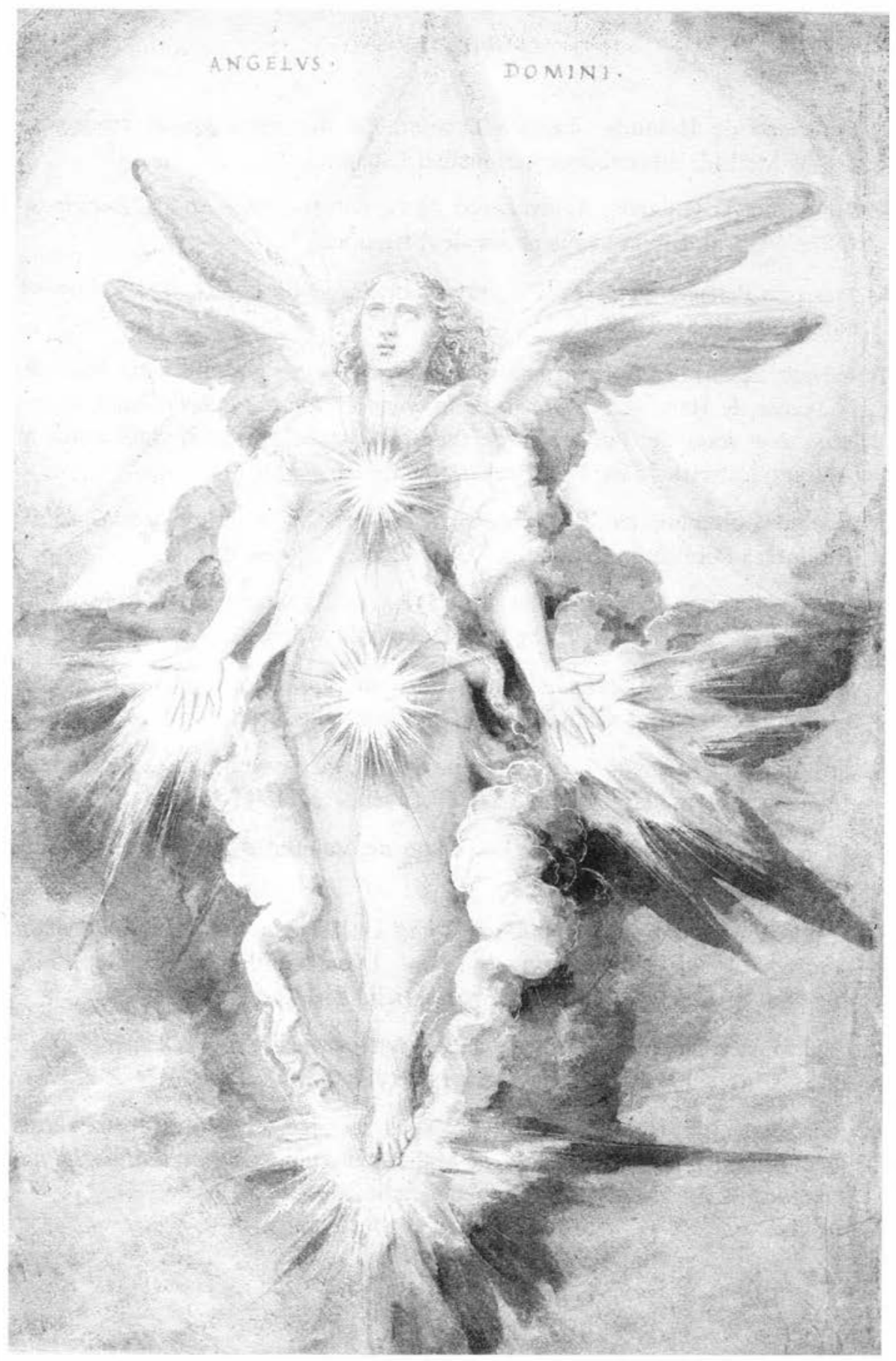

ill. 1 


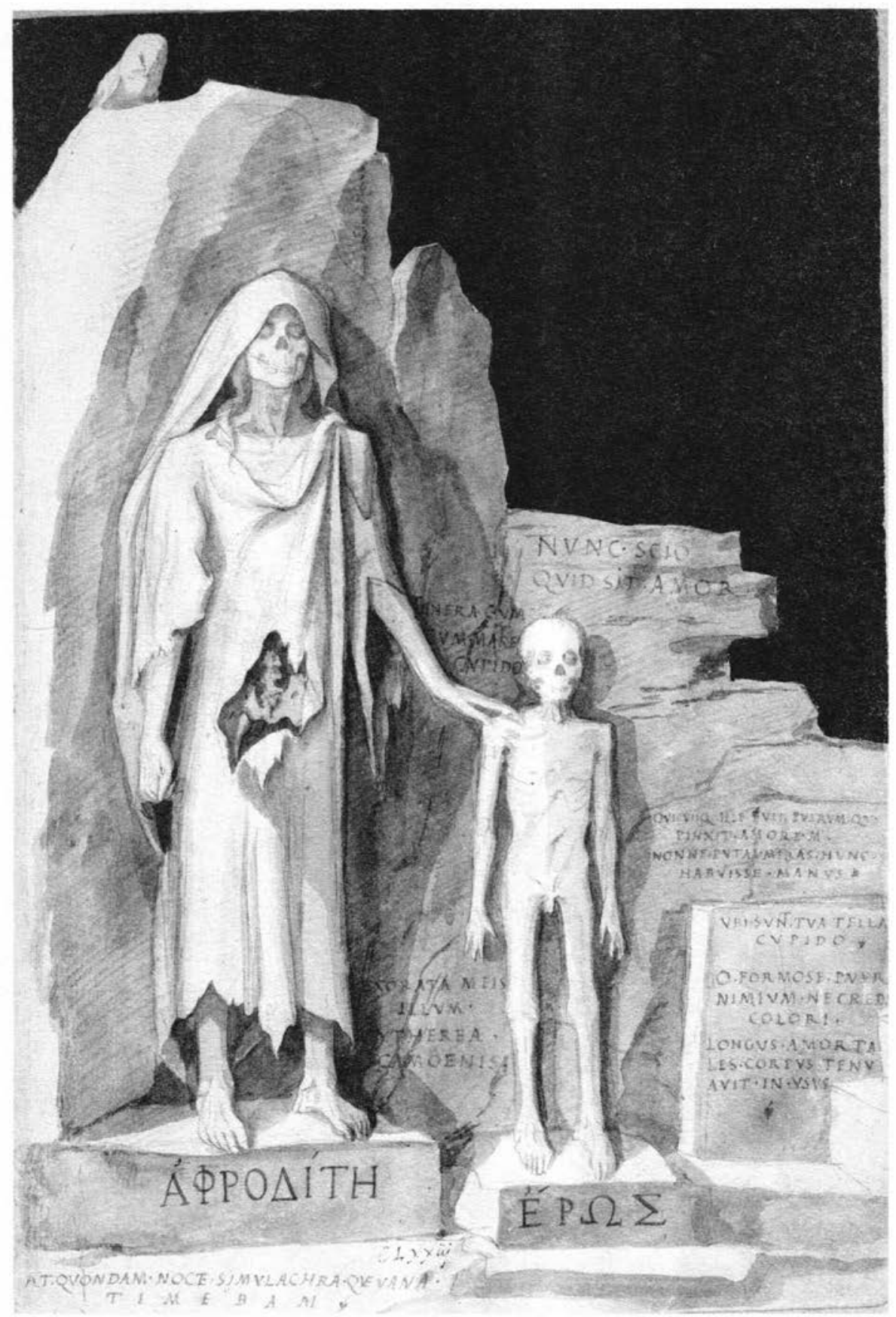

ill. 2 


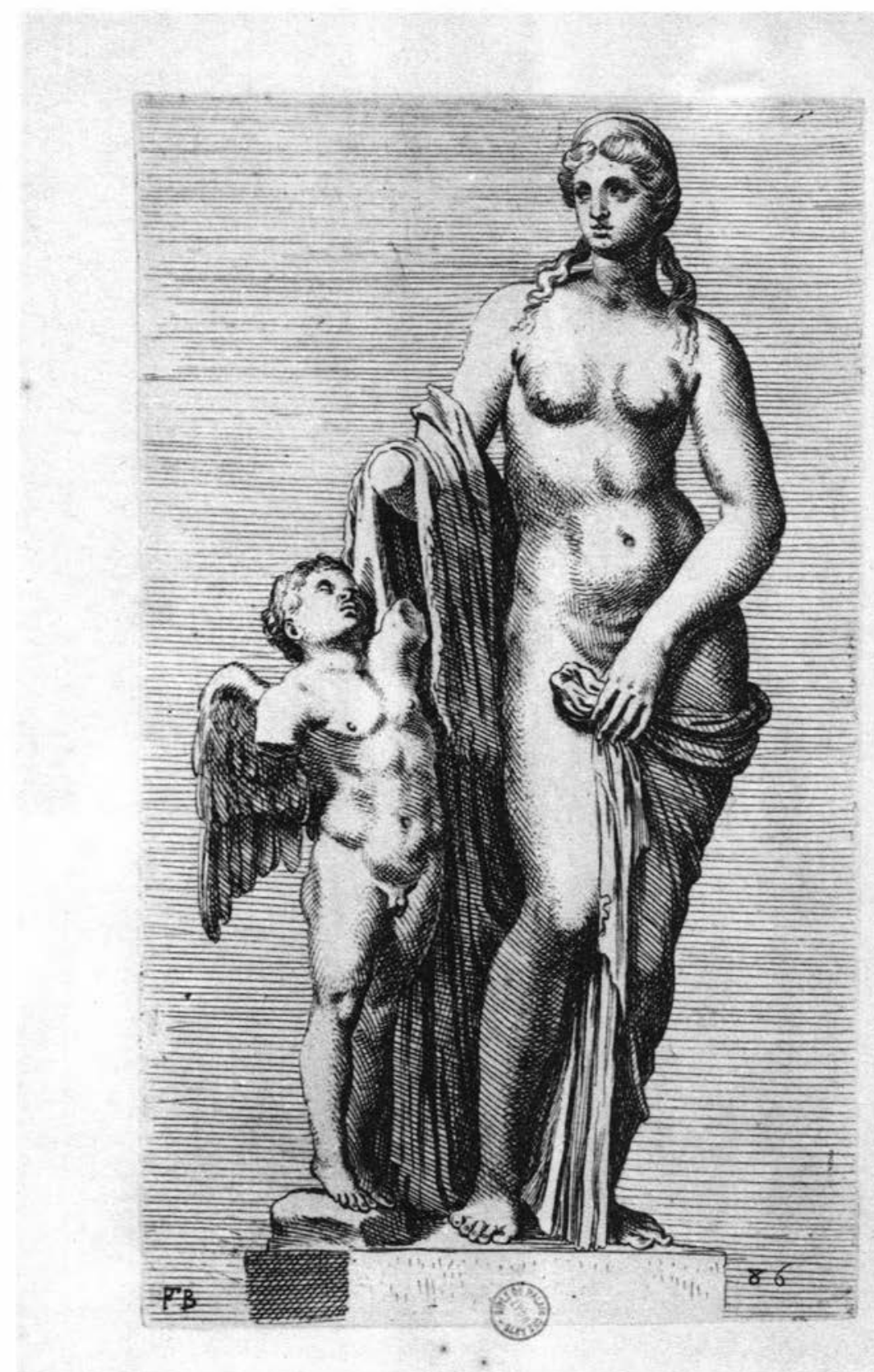

ill. 3 
TAB, PRIMERA DEL LIB, SEGVNDO

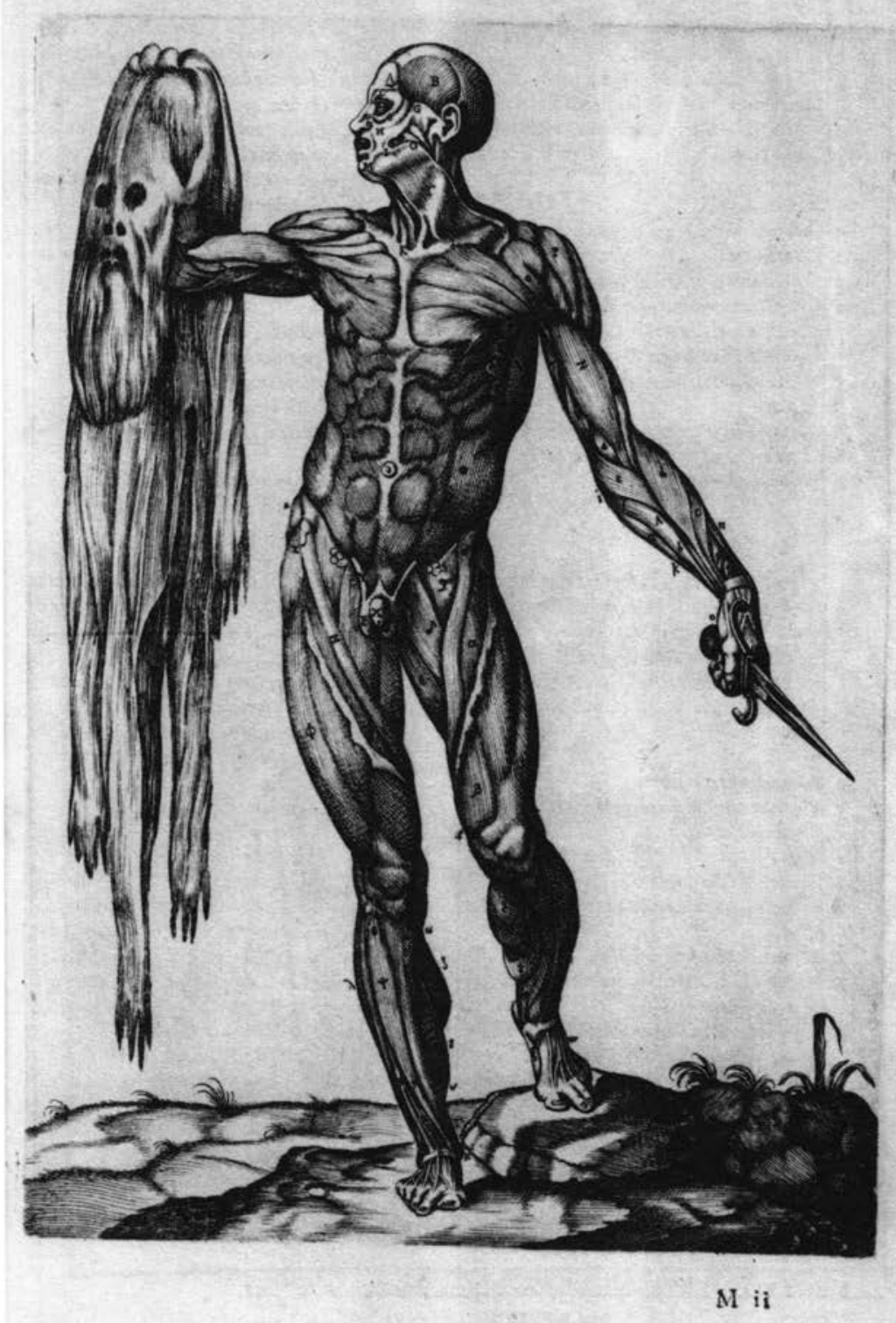

ill. 4 


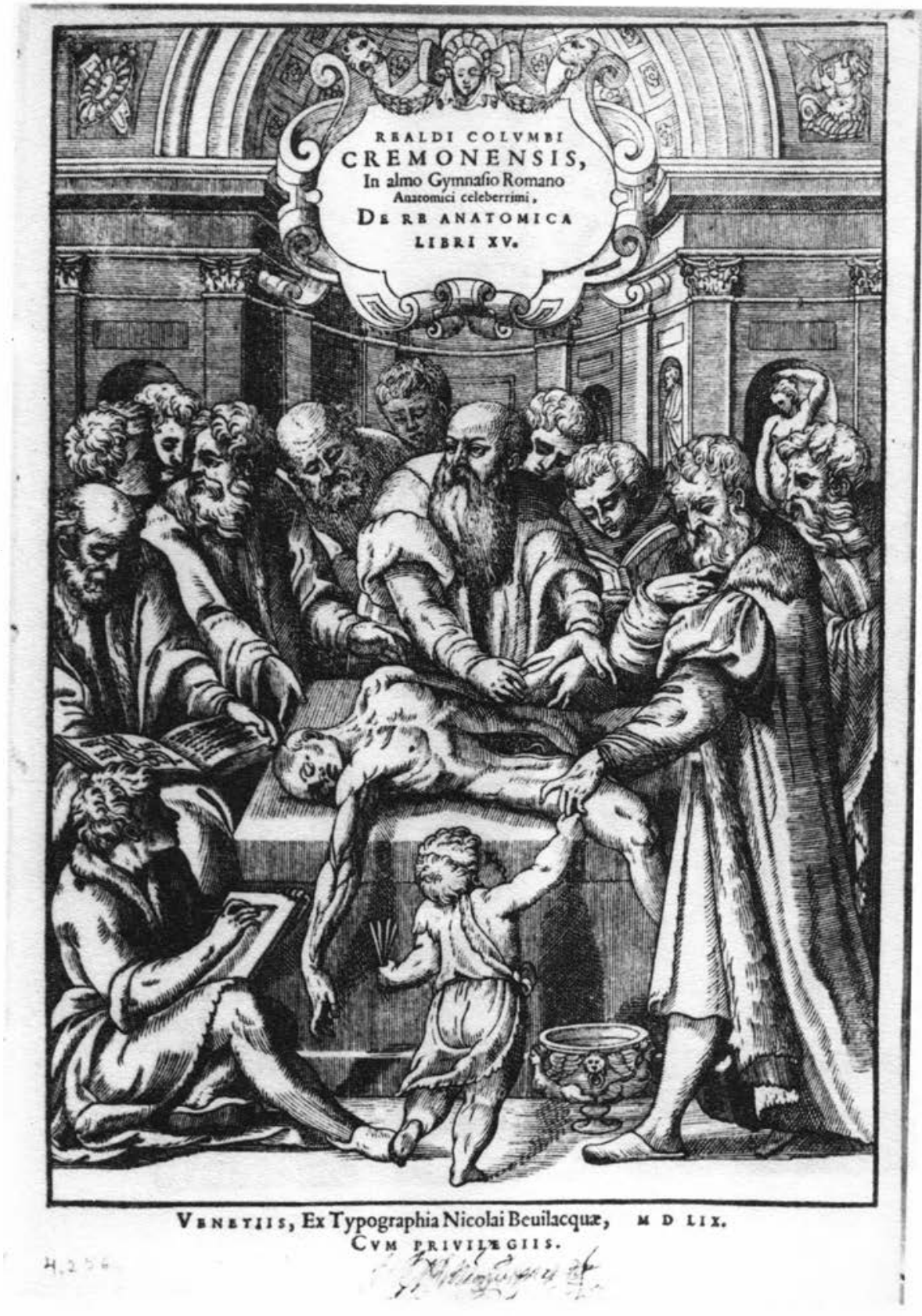

ill. 5 


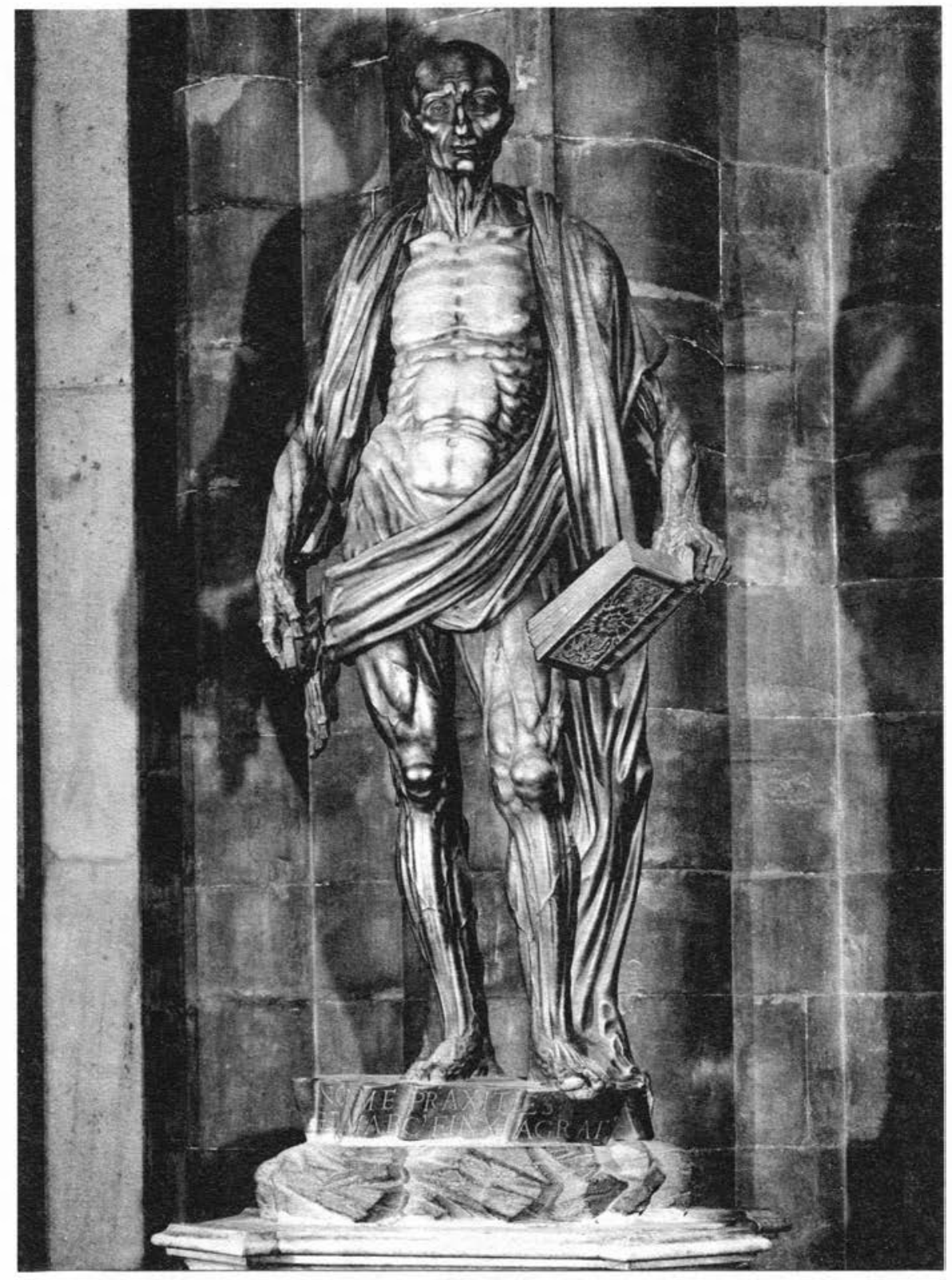

ill. 6 


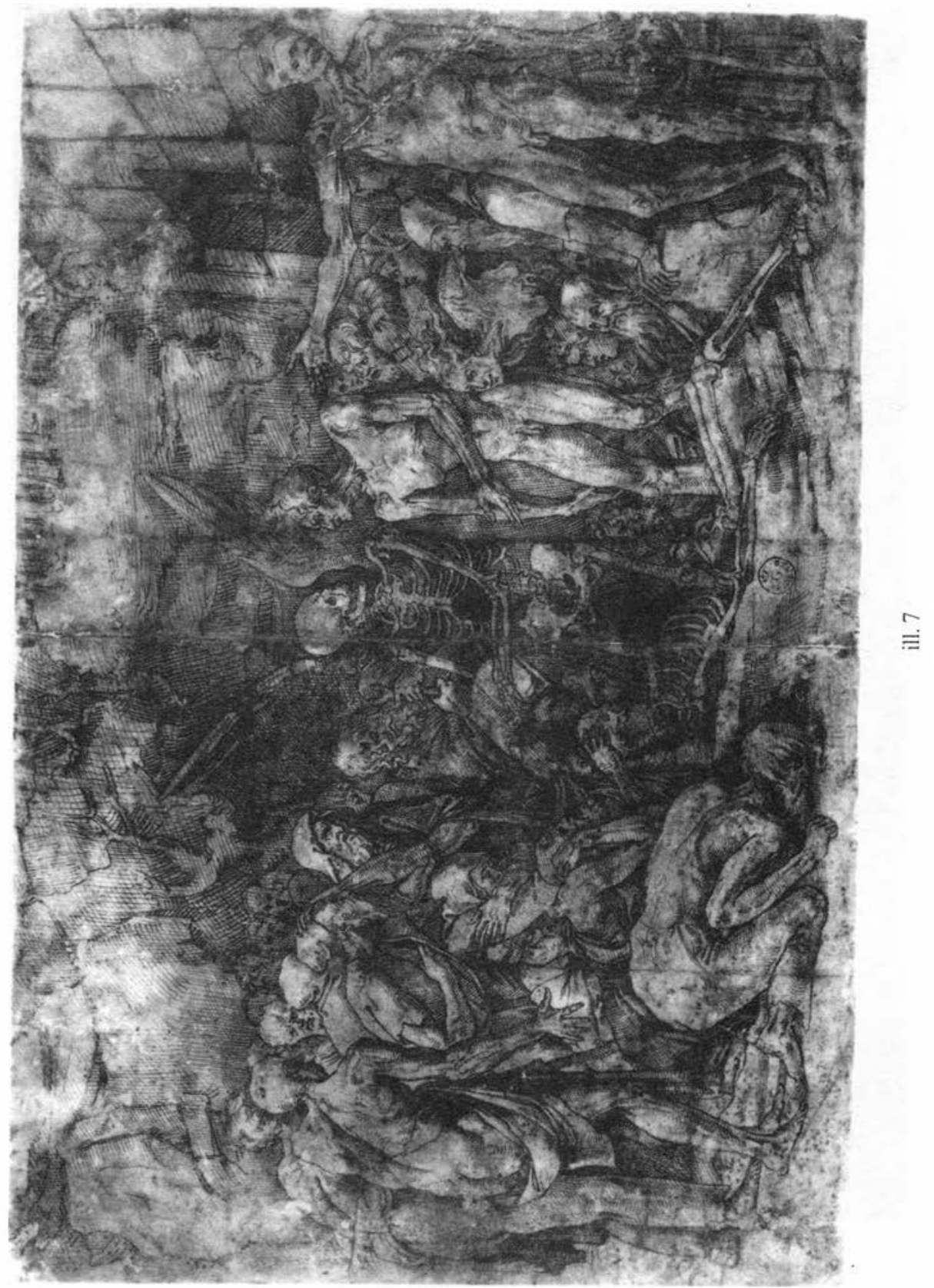




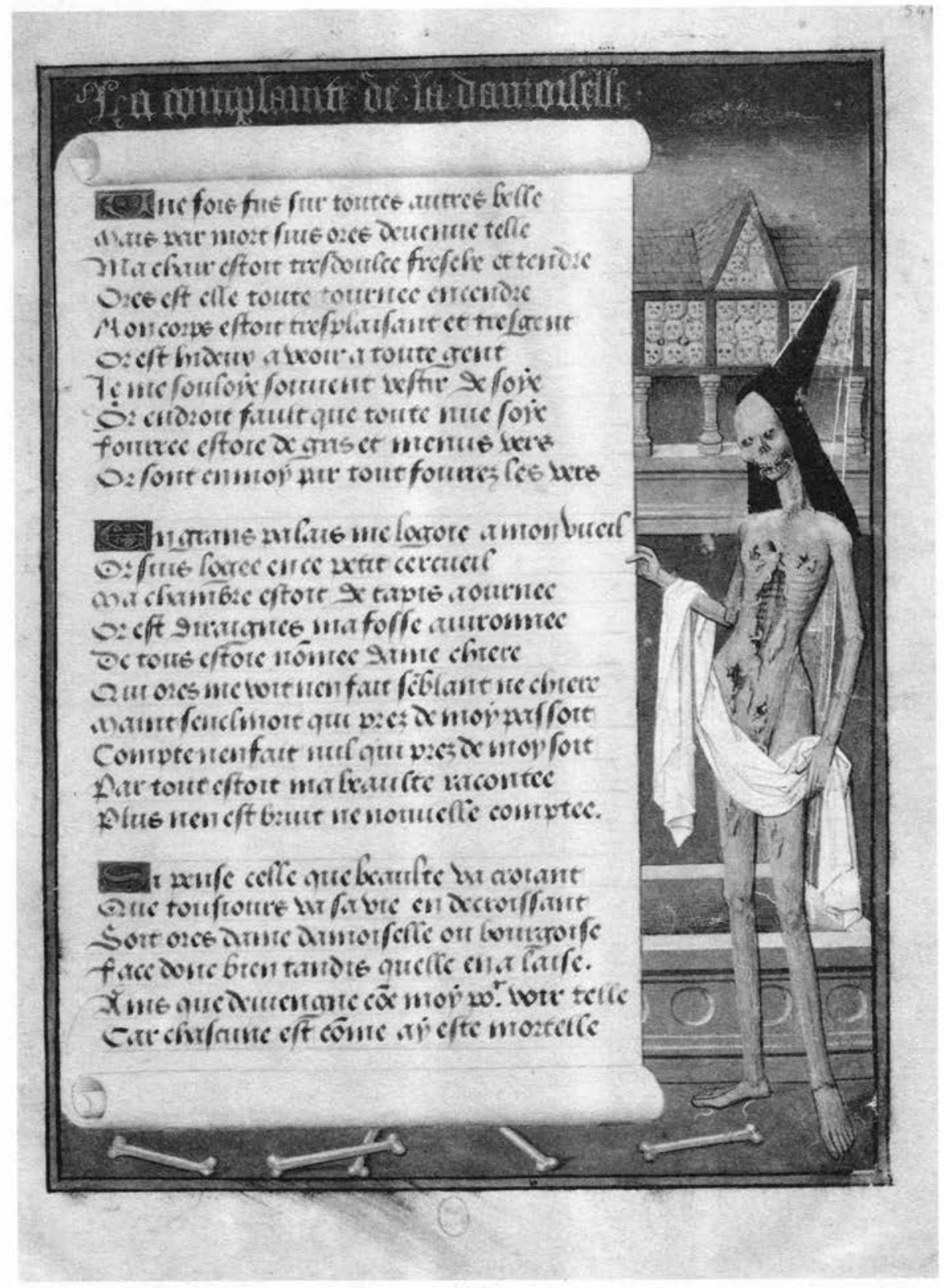

ill. 8 


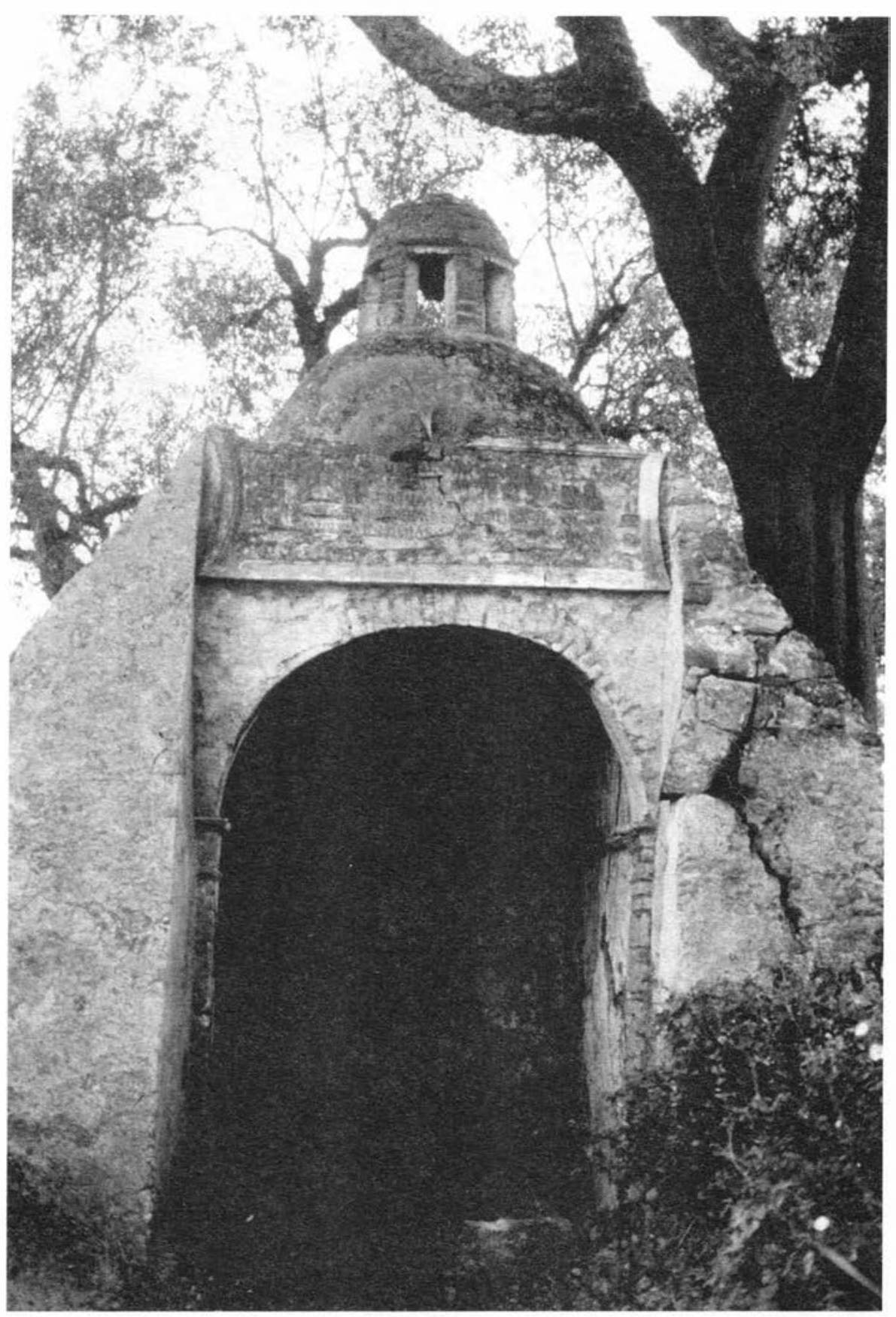

ill. 9 


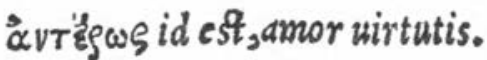

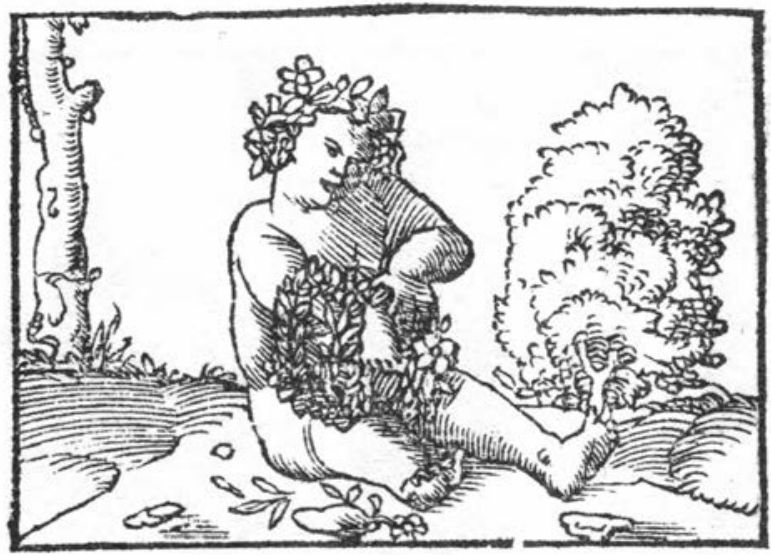

Dic ubi funt inchrui arcus?ubi te!a Cupido?

Mothia queis iunenum figere corda foles. Fax ubi triftis? ubi penne?tres unde corollas

Fert manus?unde aliam tempora cincta gerunt? Hawd mibi unlgari est, bopes cü Cypride quicquam villa uoluptatis nos neque forma tuiti. sed puris bominum fuccendo mentibus ignes

Difcipline, animos aftrág; ad alts trabo. Quatuor éf; ip a texo uirtute corollas, Qusarum que Sophie eft, tempora prims tegit. 


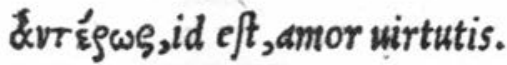

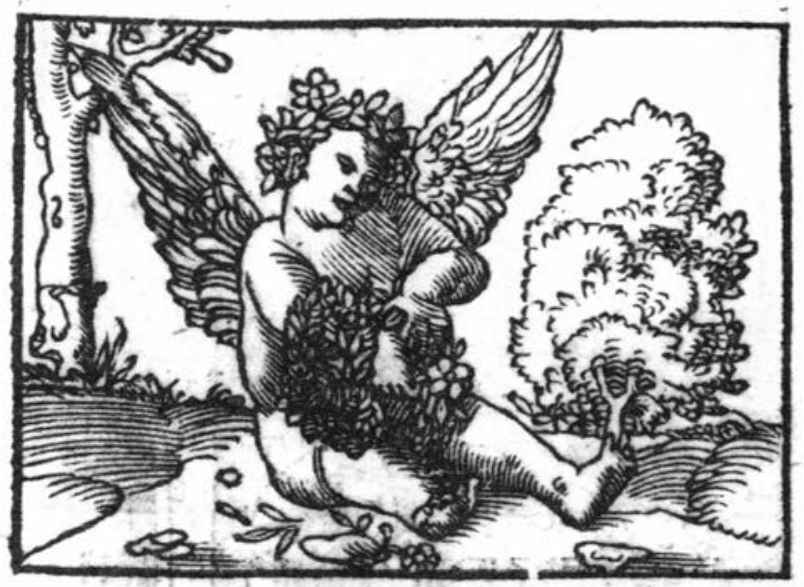

Dic ubi funt incurui arcus ? ubi tela Cupido ? Mollia queis imenum figere corda foles. \&ax ubi tristisiabi penne? tres unde corollas

Fert manus? unde divam tempord cincta gerunt? Haud mibi unigari eft, hoppes, cü cypride quicqu Vllaygluptatis, nos neque forma tulit. sed puris bominum fuccendo mencibus ignes Difcipline, enimos aftrag; ad alta trabo. Quattuor eq́j ip $\int$ d texo virtute corollas, Quarum gua sophie eftstempora prima tegit. 


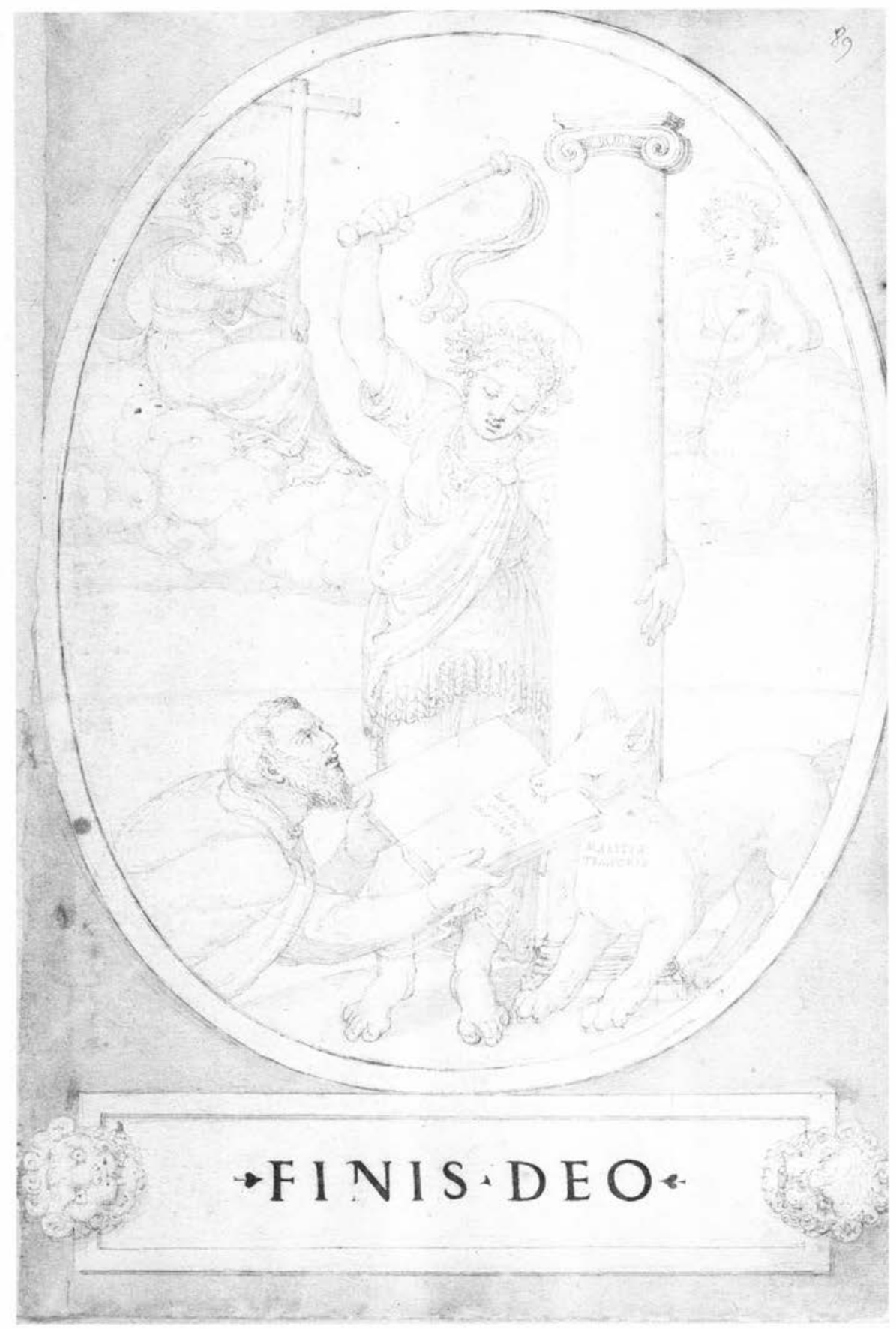

ill. 12 